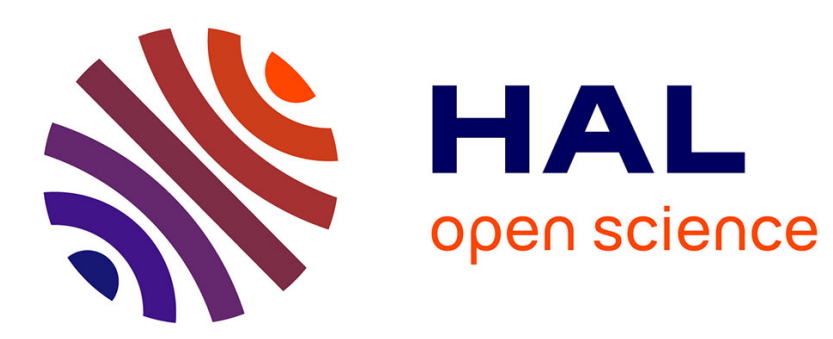

\title{
To grow or not to grow? Intermediate and paratenic hosts as helminth life cycle strategies
}

\author{
G.A. Parker, M.A. Ball, J.C. Chubb
}

\section{To cite this version:}

G.A. Parker, M.A. Ball, J.C. Chubb. To grow or not to grow? Intermediate and paratenic hosts as helminth life cycle strategies. Journal of Theoretical Biology, 2009, 258 (1), pp.135. 10.1016/j.jtbi.2009.01.016 . hal-00554564

\section{HAL Id: hal-00554564 \\ https://hal.science/hal-00554564}

Submitted on 11 Jan 2011

HAL is a multi-disciplinary open access archive for the deposit and dissemination of scientific research documents, whether they are published or not. The documents may come from teaching and research institutions in France or abroad, or from public or private research centers.
L'archive ouverte pluridisciplinaire HAL, est destinée au dépôt et à la diffusion de documents scientifiques de niveau recherche, publiés ou non, émanant des établissements d'enseignement et de recherche français ou étrangers, des laboratoires publics ou privés. 


\section{Author's Accepted Manuscript}

To grow or not to grow? Intermediate and paratenic hosts as helminth life cycle strategies

G.A. Parker, M.A. Ball, J.C. Chubb

PII:

S0022-5193(09)00007-1

DOI: doi:10.1016/j.jtbi.2009.01.016

Reference: YJTBI 5424

To appear in: $\quad$ Journal of Theoretical Biology

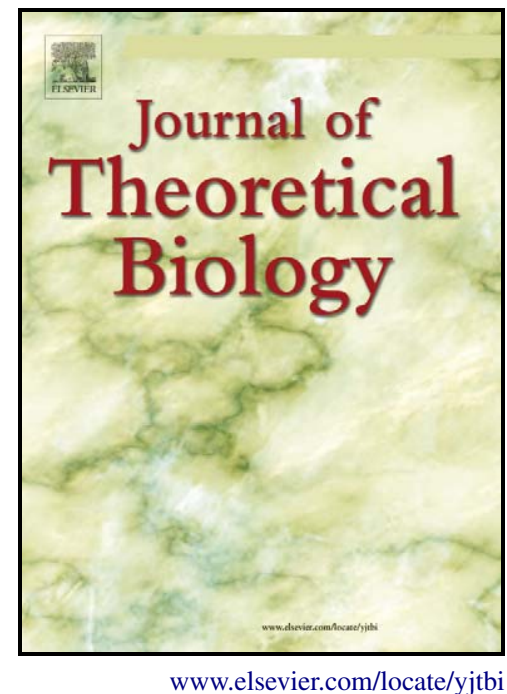

Received date: $\quad 13$ November 2008

Revised date: 16 December 2008

Accepted date: $\quad 7$ January 2009

Cite this article as: G.A. Parker, M.A. Ball and J.C. Chubb, To grow or not to grow? Intermediate and paratenic hosts as helminth life cycle strategies, Journal of Theoretical Biology (2009), doi:10.1016/j.jtbi.2009.01.016

This is a PDF file of an unedited manuscript that has been accepted for publication. As a service to our customers we are providing this early version of the manuscript. The manuscript will undergo copyediting, typesetting, and review of the resulting galley proof before it is published in its final citable form. Please note that during the production process errors may be discovered which could affect the content, and all legal disclaimers that apply to the journal pertain. 


\title{
To grow or not to grow? Intermediate and paratenic hosts as helminth life cycle strategies
}

\author{
G. A. Parker ${ }^{1}$, M. A. Ball ${ }^{2}$ \& J. C. Chubb ${ }^{1}$ \\ ${ }^{1}$ Division of Population and Evolutionary Biology, School of Biological Sciences, \\ University of Liverpool, Liverpool L69 7ZB \\ ${ }^{2}$ Division of Applied Mathematics, Department of Mathematical Sciences, University of \\ Liverpool, Liverpool L69 3BX
}

\begin{abstract}
Larval helminths in intermediate hosts often stop growing long before their growth is limited by host resources, and do not grow at all in paratenic hosts. We develop our model (Ball et al. 2008. The evolution of complex life cycles when parasite mortality is size- or time-dependent. J. Theor. Biol. 253, 202-214) for optimal growth arrest at larval maturity (GALM) in trophically-transmitted helminths. This model assumes that on entering an intermediate host, larval death rate initially has both time- (or size-) dependent and time-constant components, the former increasing as the larva grows. At GALM, mortality changes to a new and constant rate in which the size-dependent component is proportional to that immediately before GALM. Mortality then remains constant until death or transmission to the definitive host. We analyse linear-increasing and accelerating forms for time-dependent mortality to deduce why there is sometimes growth (intermediate hosts) and sometimes no growth (paratenic hosts). Calling $\mathrm{i}$ the intermediate or paratenic host, and $\mathrm{j}$ the definitive host, conditions favouring paratenicity are: (i) high values in host i for size at establishment, size-related mortality, expected intensity, (ii) low values in host i for size-independent mortality rate, potential growth rate, transmission rate to $j$, and ratio of death rate in $j$ /growth rate in $j$. Opposite conditions favour growth in the (intermediate) host, either to GALM, or until death without GALM. We offer circumstantial evidence from the literature supporting some of these predictions. In certain conditions, two of the three possible growth strategies (no growth; growth to an optimal size then growth arrest (GALM); unlimited growth until larval death) can coexist as local optima. The effect of the discontinuity in death rate after GALM is complex and depends on mortality and growth parameters in the two hosts, and on the mortality functions before and after GALM.
\end{abstract}

Keywords: Helminth evolution; life history strategies; growth arrest of larvae; intermediate hosts; paratenic hosts 


\section{Introduction}

The present paper and its companion (Ball, Chubb \& Parker, 2008; hereafter BPC) extend the logic of evolution of complex helminth life cycles by replacing the assumption of constant mortality of larval parasites (Parker et al., 2003a) with the more realistic supposition that mortality increases with time in the intermediate host, e.g. because of increases in parasite mass or host responses.

Our models are for complex life cycles in which the helminth passes through at least one intermediate host (i.e. a host in which there is growth but no sexual reproduction) before transmission to the definitive host (i.e. a host in which there is sexual maturation and release of propagules). We specifically analyse the case where parasites are transferred in the food of their host (trophic transmission), e.g. when a predator consumes prey containing helminth larvae, as is typically the case in nematodes, cestodes and acanthocephalans. Iwasa and Wada (2006) have modelled the case where larvae leave an intermediate host to seek their definitive host, as applies in many trematodes. They show that a constant, random death rate of the larva in the intermediate host can readily generate an optimal size at which the larva should leave its host to search for the definitive host, exactly as a constant death rate can tune the switch from growth to sexual maturity in the definitive host. By contrast, in trophic transmission, the helminth larva must await ingestion of the intermediate host by the definitive host. Here, constant larval mortality in the intermediate host does not generate an optimal size at which the larva should stop growing in that host, though such an optimum can be generated if the larval mortality rate increases with time spent in the intermediate host (BPC). 
BPC defined the age (or size) at which a helminth larva with trophic transmission should optimally undergo growth arrest at larval maturity (GALM) in an intermediate host (see also Parker et al., 2003b). Here, we develop BPC's general equation for the size (or age) at which larvae should optimally stop growing, and examine the biology of larval growth strategies in the light of its predictions. For simplicity, we confine our analysis to larval growth in the host preceding the definitive host, following BPC. We are especially interested in the evolution of the parasitological phenomenon of paratenicity; i.e. how to explain the difference between hosts in which larvae grow (intermediate hosts), and those hosts in which larvae do not grow (paratenic hosts).

Paratenic hosts are typically defined as hosts that occur at some stage before the definitive host, but in which the parasite shows no apparent growth or development (Bush et al., 2001). They are often explained away as incidental transport hosts or 'ecological bridges' that enhance transmission between hosts in a life cycle (e.g. Marcogliese, 2001). However, intermediate hosts also have a transmission function: the fundamental distinction between intermediate and paratenic hosts relates to the presence or absence of larval growth and development. We thus argue that it is the parasite's life history strategy that defines the difference between intermediate and paratenic hosts, and here predict conditions likely to generate paratenicity. BPC have shown that conditions determining size (or age) at GALM are independent of the pathway by which a complex life cycle evolves, and are influenced only by the growth and mortality parameters for the larva in its hosts. 


\section{Analysis}

\subsection{The general equation for GALM}

Parker et al. (2003a) defined two components of mortality for a parasite exploiting a given host species $\mathrm{i}$, and for which species $\mathrm{j}$ represents a potential new host higher up the food chain. 'Noise mortality' is the death rate of the parasite that is unrelated to $j$, and 'transmission mortality' is its death rate due to ingestion by $\mathrm{j}$. When $\mathrm{j}$ becomes incorporated into the parasite life cycle as a new host, transmission mortality is converted into the transmission rate to host $\mathrm{j}$. Noise mortality relates to parasite death, not host death, unless they are synonymous.

Consider a complex cycle in which $\mathrm{i}$ is the intermediate and $\mathrm{j}$ the definitive host; time $t=0$ is the time of entry of the free-living propagule into host $\mathrm{i}$. BPC proposed that larval noise mortality in host $\mathrm{i}$ would be time-dependent, and discontinuous at time $t_{d}$, when the larva stops growing (GALM) at size $W_{d}$. Thus time $t_{d}$ divides the parasite's residency in host $\mathrm{i}$ into two phases (see Fig. 1). The first phase applies during active growth and development $\left(0 \leq t \leq t_{d}\right)$, and noise mortality rate, $p_{n i}^{(1)}\left(t \leq t_{d}\right)$, is assumed to increase with the size (or age) of the larva. The second phase applies after GALM, when noise mortality is assumed to remain constant with time at rate $p_{n i}^{(2)}\left(t_{d}\right)$, and dependent only on the larval size (or age) at GALM. We assume that the larva enters host $\mathrm{i}$ at size $W_{0}$, and there is a maximum larval size, $W_{m}$, at which the mortality rate becomes infinite (see also $\mathrm{BPC})$. 
Biologically it seems most likely for vertebrate hosts that typically, the size- or timedependent noise mortality reduces at GALM as in Figs. 1a, 1c (see BPC and Discussion), but we allow that for some systems there could be a small increase in mortality at GALM as in Figs. 1b, 1d. From the parasite's perspective, the lowest value for noise mortality after GALM yields the highest potential fitness benefits.

Calling $p_{i j}$ the transmission rate from the intermediate host $\mathrm{i}$ to the definitive host $\mathrm{j}$ (proportional to the rate at which $\mathrm{i}$ is consumed by $\mathrm{j}$ ), $p_{j}$ the (constant) noise mortality rate in host $\mathrm{j}$, and $g_{i}, g_{j}$ the growth rates in $\mathrm{i}$ and $\mathrm{j}$ respectively, BPC showed that at the optimal time $\left(t_{d}^{*}\right)$ and size $\left(W_{d}^{*}\right)$ for GALM in host $\mathrm{i}$, the following equation applies if growth is linear and birth rate is proportion to body mass:

$p_{n i}^{(1)}\left(t_{d}^{*}\right)-p_{n i}^{(2)}\left(t_{d}^{*}\right)=p_{j} g_{i} / g_{j}-\left(d p_{n i}^{(2)} / d t_{d}\right) /\left(p_{n i}^{(2)}\left(t_{d}^{*}\right)+p_{i j}\right)$.

Eq. (1) was obtained by taking parasite fitness (expected number of eggs) when GALM occurs at time $t_{d}$ away from the fitness expected at time $t_{d}+\delta t_{d}\left(\delta t_{d}\right.$ small), and dividing by $\delta t_{d}$. This has the same result as taking the derivative with respect to $t_{d}$, which must be zero at the maximum value for fitness.

A brief general interpretation of Eq. (1) is useful. The left hand side of Eq. (1) is the change in mortality rate at GALM (time $\left.t_{d}^{*}\right)$. We argue that typically $p_{n i}^{(1)}\left(t_{d}^{*}\right)>p_{n i}^{(2)}\left(t_{d}^{*}\right)$, i.e. mortality immediately after GALM will be less than that before GALM, since active growth has ceased (see Fig. 1a, 1c). However, we also examine instances in which $p_{n i}^{(2)}\left(t_{d}^{*}\right)>p_{n i}^{(1)}\left(t_{d}^{*}\right)$, i.e. mortality increases at GALM (see Fig. 1b, 1d). In the biologically plausible cases we analyse the magnitude of the change, $p_{n i}^{(1)}\left(t_{d}^{*}\right)-p_{n i}^{(2)}\left(t_{d}^{*}\right)$, increases with $t_{d}$ (Fig. 1). Now, for an intermediate optimal size at GALM (i.e. $W_{0}<W_{d}^{*}<W_{m}$ ), the 
mortality change at GALM must balance the right hand side of Eq. (1), which consists of the positive constant term $\left(p_{j} g_{i} / g_{j}\right)$ minus the term $\left(d p_{n i}^{(2)} / d t_{d}\right) /\left(p_{n i}^{(2)}\left(t_{d}^{*}\right)+p_{i j}\right)$. This latter term changes with the gradient $d p_{n i}^{(2)} / d t_{d}$ divided by the absolute value of $p_{n i}^{(2)}\left(t_{d}\right)$, and may be either increasing or decreasing with $t_{d}$, depending on the form of $p_{n i}^{(2)}\left(t_{d}\right)$.

For Eq. (1) to give a maximum and not a minimum, we need $d p_{n i}^{(2)} / d t_{d}-\left(d^{2} p_{n i}^{(2)} / d t_{d}^{2}\right) /\left(p_{n i}^{(2)}+p_{i j}\right)+\left(d p_{n i}^{(2)} / d t_{d}\right)^{2} /\left(p_{n i}^{(2)}+p_{i j}\right)^{2}-d p_{n i}^{(1)} / d t_{d}<0$

For the strategy of paratenicity (no growth) in host $i$ to be a local maximum, the first derivative of the fitness function with respect to time $t_{d}$ must be less than zero at time $t_{d}=0$, i.e. the parasite remains at its entry size, $W_{0}$. Thus

$p_{n i}^{(2)}(0)+p_{j} g_{i} / g_{j}-\left(d p_{n i}^{(2)}(0) / d t_{d}\right) /\left(p_{n i}^{(2)}+p_{i j}\right)-p_{n i}^{(1)}(0)<0$

To check the solutions generated from Eq. (1) for $W_{d}^{*}$, we first established that they were maxima using condition (2a); if this was violated we iterated fitness in relation to $t_{d}$ to determine whether a fitness maximum occurs at limit $W_{0}$ or $W_{m}$, or whether both extremes can be local optima (see Appendix A). For paratenicity, we also ensured that condition (2b) applied.

BPC derived Eq. (1) with a single parasite in mind (i.e. an intensity of one), but from unpublished analyses under restricted assumptions, concluded that $W_{d}^{*}$ would approximate to the total mass of all larvae in host i, i.e. for an intensity of $N$, the average mass of each larva would be roughly $W_{d}^{*} / N$. For our present analysis, we also assume an intensity of $N=1$ unless otherwise stated.

\subsection{Application of explicit functions for $p_{n i}^{(1)}$ and $p_{n i}^{(2)}$}


We assume that during active growth (i.e. at times $0 \leq t \leq t_{d}$ ), larval mortality rate $p_{n i}^{(1)}\left(t<t_{d}\right)$ increases continuously with larval size, $W$. Although we focus on sizedependent mortality, this analysis relates time and size linearly and our conclusions thus apply to any process where mortality rates increase with time. After growth arrest, larval mortality rate remains constant at $p_{n i}^{(2)}\left(t=t_{d}\right)$ (see Fig. 1).

To gain a feel for how growth is likely to vary depending on the conditions faced by a larval helminth in its intermediate host, we must apply explicit forms of $p_{n i}^{(1)}$ and $p_{n i}^{(2)}$ to Eq. (1). BPC assumed that the noise mortality rate during growth, $p_{n i}^{(1)}$, has a constant, time-independent component, $p_{n i}^{0}$ (e.g. which includes the predation rate on the host by predators other than the definitive host), and an increasing, time-dependent component, $M(t)$. We investigate two simple increasing forms for $M(t)$ : (i) linear, up to a size $W_{m}$, after which the rate suddenly becomes infinite, and (ii) accelerating, which increases continuously up to infinity at size $W_{m}$. For $p_{n i}^{(2)}$, we assume that at GALM, the time-independent component, $p_{n i}^{0}$, remains unchanged, but the time-dependent component remains constant with time at the value $\alpha M\left(t_{d}\right)$, in which $\alpha$ becomes scaling parameter. For illustrative purposes, we investigate cases when $\alpha$ is a constant.

For simplicity, Eq. (1) assumes a linear relation between size and time, thus in host $\mathrm{i}$ : $W(t)=W_{0}+g_{i} t$ (see also Parker et al., 2003a; BPC). The most typical form of growth is probably logistic, as used by Iwasa and Wada (2006) in their analysis of complex life cycles of helminths that transmit from one host to the next by active larval migration, such as trematodes. However, different stages of the same helminth species may have contrasting forms of growth. For instance, in Schistocephalus solidus, a helminth for 
which there is good data, growth rate is decreasing in the first host (Michaud et al., 2006) and (at least initially) increasing in the second host (e.g. Sharsack et al., 2007; Clarke, 1954). Since our aims in this paper and its companion (BPC) are heuristic (i.e. to show the sorts of solution that can exist, rather than to make predictions for given species), the assumption of linear growth is justified in the interests of mathematical tractability. To apply our model to a given species, we would obviously wish to derive and use the appropriate relationships and parameters for that species in order to make quantitative predictions.

Some of our parameters could arguably be functions of time $t$. For instance, although function $p_{n i}^{(2)}(t)$ may typically be constant for a long time after GALM, it must eventually increase, if for no other reason that host i must occasionally die through senescence, rather than remain constant as in our models. Increased parasite size may possibly change transmission rate, $p_{i j}$, making $p_{i j}$ a function of $t$. Note also that we consider only growth strategies in host $\mathrm{i}$, the host immediately before the definitive host $\mathrm{j}$. Cycles with two intermediate hosts, $\mathrm{h}$ and $\mathrm{i}$, are not uncommon in helminths, and we would require a different analysis for optimal growth in host $\mathrm{h}$. We avoid all such complications to preserve simplicity in an already complex model.

A glossary of mathematical terms is given in BPC.

\subsection{Mortality before GALM, $p_{n i}^{(1)}(t)$}


Where $p_{n i}^{g}$ is a constant scaling the size- or time-dependent component of noise mortality rate of the parasite in host $\mathrm{i}$, the two explicit forms for noise mortality before GALM (Fig. 1) investigated are:

(i) Linear increasing

$p_{n i L}^{(1)}(t)=p_{n i}^{0}+p_{n i}^{g}\left(W_{0}+g_{i} t\right), W<W_{m}$,

$p_{n i L}^{(1)}(t)=\infty, W \geq W_{m}$

(ii) Accelerating

$p_{n i A}^{(1)}(t)=p_{n i}^{0}+p_{n i}^{g}\left(W_{0}+\frac{\left(W_{m}-W_{0}\right)^{2}}{\left(W_{m}-\left(W_{0}+g_{i} t\right)\right)}-\left(W_{m}-W_{0}\right)\right), W<W_{m}$,

$p_{n i A}^{(1)}(t)=\infty, W \geq W_{m}$

Thus in both forms, noise mortality rate is the sum of the constant, time-independent mortality rate, $p_{n i}^{0}$, and the size-dependent rate which increases with parasite size or age from $p_{n i}^{g} W_{0}$ at the moment of establishment, time $t=0$ (see continuous lines up to the open circles in Fig. 1). They also have the same initial rate of increase, i.e.

$d p_{n i}^{(1)}(0) / d t_{d}=p_{n i}^{g} g_{i}$, but while the linear form increases at constant rate, the accelerating form increases at a rate that increases with $t$. Note that we have modified the accelerating form suggested by BPC so that the two forms have the same values and gradients at time $t=0$.

Our original treatment of noise mortality (Parker et al., 2003a) included only the constant, time-independent component, $p_{n i}^{0}$, and assumed that the parasite died if it reached size $W_{m}$. Further interpretations of the noise mortality functions and constant $p_{n i}^{g}$ are given in BPC.

Applying (1), linear mortality Eq. (3) gives

$W_{d}^{*}=E / p_{n i}^{g}$, 
and accelerating mortality Eq. (4) gives

$W_{d}^{*}=W_{m}-\left(W_{m}-W_{0}\right)^{2} /\left(E / p_{n i}^{g}+W_{m}-2 W_{0}\right)$,

where $E=p_{n i}^{(2)}\left(t_{d}^{*}\right)-p_{n i}^{0}+p_{j} g_{i} / g_{j}-\left(d p_{n i}^{(2)} / d t_{d}\right) /\left(p_{n i}^{(2)}\left(t_{d}^{*}\right)+p_{i j}\right)$, with $p_{n i}^{(2)}=p_{n i L}^{(2)}, p_{n i A}^{(2)}$ for

the linear and accelerating mortality models respectively. Note that both behave similarly: in each, $W_{d}^{*}$ increases with $E / p_{n i}^{g}$.

2.4. Mortality after GALM, $p_{n i}^{(2)}\left(t_{d}\right)$

We assume that both the time-independent and time-dependent components of noise mortality remain constant with time after GALM $\left(t>t_{d}\right)$, as $p_{n i}^{0}$ (the dotted lines in Fig. 1) and $p_{n i}^{(2)}\left(t_{d}\right)$ (the continuous lines after the filled circles in Fig. 1). However, since we assume that the size-dependent component of noise mortality after GALM is proportional to that immediately before GALM, $p_{n i}^{(2)}\left(t_{d}\right)$ increases with the time, $t_{d}$, at which GALM occurs (see broken lines in Fig. 1). Thus

(i) Linear increasing

$p_{n i L}^{(2)}\left(t_{d}\right)=p_{n i}^{0}+\alpha p_{n i}^{g}\left(W_{0}+g t_{d}\right)$,

following Eq. (3). Hence $d p_{n i L}^{(2)} / d t_{d}=\alpha p_{n i}^{g} g_{i}$ and $d^{2} p_{n i L}^{(2)} / d t_{d}^{2}=0$.

(ii) Accelerating

$p_{n i A}^{(2)}\left(t_{d}\right)=p_{n i}^{0}+\alpha p_{n i}^{g}\left(W_{0}+\frac{\left(W_{m}-W_{0}\right)^{2}}{\left(W_{m}-\left(W_{0}+g_{i} t_{d}\right)\right)}-\left(W_{m}-W_{0}\right)\right)$,

following Eq. (4). Hence $d p_{n i A}^{(2)} / d t_{d}=\alpha p_{n i}^{g} g_{i}\left(W_{m}-W_{0}\right)^{2} /\left(W_{m}-W_{d}\right)^{2}$ and $d^{2} p_{n i A}^{(2)} / d t_{d}^{2}=2 \alpha p_{n i}^{g} g_{i}^{2}\left(W_{m}-W_{0}\right)^{2} /\left(W_{m}-W_{d}\right)^{3}$. 


\subsection{Solutions}

\section{(i) Linear increasing}

Substituting the linear forms Eqs. (3) and (7) into Eq. (1) generates a quadratic equation for the optimal size at GALM, $W_{d}^{*}$. Calling $\beta=p_{j} g_{i} / g_{j}$, and $\gamma=p_{n i}^{0}+p_{i j}$, the coefficients are:

$a=\alpha p_{n i}^{g}(1-\alpha)$

$b=[(1-\alpha) \gamma-\alpha \beta]$,

$c=\alpha g_{i}-\beta \gamma / p_{n i}^{g}$.

We require the positive root, subject (from condition $(2 \mathrm{a})$ ) to

$\alpha^{2} p_{n i}^{g} g_{i} /\left[\gamma+\alpha p_{n i}^{g} g_{i}\left(W_{0}+g_{i} t_{d}^{*}\right)\right]^{2}<(1-\alpha)$

for a maximum. Note that if noise mortality increases at GALM (i.e. $\alpha>1$ ), condition (9) cannot be satisfied; a non-intermediate solution for growth (i.e. $W_{0}$ or $W_{m}$ ) is always the optimal strategy.

(ii) Accelerating

Calling $W_{y}=W_{m}-W_{0}$, and $W_{z}^{*}=W_{m}-W_{d}^{*}$, and substituting Eqs. (4) and (8) into Eq. (1), we can generate a quadratic equation for $W_{z}^{*}$, from which we can calculate the optimal size at GALM, $W_{d}^{*}$. The quadratic solution for $W_{z}^{*}$ now has coefficients:

$$
\begin{aligned}
& a=\left[\gamma+\alpha p_{n i}^{g}\left(2 W_{0}-W_{m}\right)\right]\left[(1-\alpha)\left(2 W_{0}-W_{m}\right)-\beta / p_{n i}^{g}\right] / W_{y}^{2}, \\
& b=\left[2 p_{n i}^{g} \alpha(1-\alpha)\left(2 W_{0}-W_{m}\right)+(1-\alpha) \gamma-\alpha \beta\right], \\
& c=\alpha\left[p_{n i}^{g}(1-\alpha) W_{y}^{2}+g_{i}\right] .
\end{aligned}
$$


We require the negative root, and setting $W_{z}^{*}=W_{m}-W_{d}^{*}$ we obtain a solution for $W_{d}^{*}$ from the above coefficients, subject to (from condition (2a)):

$\frac{\alpha}{\left[W_{z}^{*}\left(\gamma+\alpha p_{n i}^{g}\left(W_{0}+W_{y}^{2} / W_{z}^{*}-W_{y}\right)\right)\right]}\left(\frac{p_{n i}^{g} g_{i} W_{y}^{2}}{\left[W_{z}^{*}\left(\gamma+\alpha p_{n i}^{g}\left(W_{0}+W_{y}^{2} / W_{z}^{*}-W_{y}\right)\right)\right]}-2\right)<(1-\alpha)$

for a maximum. If noise mortality increases at GALM (i.e. $\alpha>1$ ), under certain parameter values condition (10) can be satisfied; unlike the linear case, an intermediate solution for larval growth can be an optimal strategy.

Since they have the same intercepts and gradients at time $t=0$, from condition (2b) both linear increasing and accelerating forms give the same requirement for paratenicity: $\frac{\beta}{p_{n i}^{g}}-\frac{\alpha g_{i}}{\left(\gamma+\alpha p_{n i}^{g} W_{0}\right)}<W_{0}(1-\alpha)$

Note that condition (11) is least likely to be satisfied if $\alpha$ is very small; if $\alpha \rightarrow 0$ condition (11) simplifies to $\beta<p_{n i}^{g} W_{0}$. If $\alpha$ is huge, condition (11) can never be satisfied. For the case where the initial size is negligible, i.e. $W_{0} \rightarrow 0$, paratenicity is locally stable if $\alpha>p_{j}\left(p_{n i}^{0}+p_{i j}\right) / p_{n i}^{g} g_{j}$

\section{Results}

The two forms for noise mortality can generate three possible optimal larval growth strategies for larvae in their intermediate host: (i) zero growth (paratenicity, with $\left.W_{d}^{*}=W_{0}\right)$, (ii) growth to an intermediate optimal size, (GALM; with $W_{0}<W_{d}^{*}<W_{m}$ ), and (iii) continuous growth until death of the parasite at or before size $W_{m}\left(W_{d}^{*}=W_{m}\right)$. 
Depending on the parameter values, all three outcomes are possible in both the linear and accelerating mortality models, and across some parameter conditions, simultaneous alternative local optima can exist. Typically, alternative local optima occur at the extremes, i.e. paratenicity or continuous growth until death, $\left(W_{0}, W_{m}\right)$, though over small regions of parameter space, intermediate growth can be an alternative to one of these extremes.

The best growth strategy for the larva in host i depends on the interaction between the immediate cost or benefit of GALM, i.e. whether it immediately reduces or increases mortality rate, and the value of host $\mathrm{j}$ relative to host $\mathrm{i}$ for growth and survival. For example, suppose that GALM offers a safe haven by reducing mortality $(\alpha<1)$, as seems biologically most likely for most cases. If host $\mathrm{j}$ is a good host in terms of low mortality and high growth rate, (low $p_{j} / g_{j}$ ), paratenicity or GALM at a low size in host $\mathrm{i}$ is favoured because risky growth in i can be exchanged for much safer growth in host $\mathrm{j}$. If host $\mathrm{j}$ offers poor survival and growth prospects relative to host i (low $p_{j} / g_{j}$ ), the reverse applies, it is safer to grow in $\mathrm{i}$ so that maturity can be attained more quickly in host $\mathrm{j}$.

One might suspect that if GALM increases mortality $(\alpha>1)$, there would be little point in the larva arresting its growth. However, provided that the costs associated with GALM are not prohibitive, these immediate costs can be much less than the mortality costs of growth later on as size increases, or the host response becomes much stronger. Encystment must have some small energetic cost, which may translate into increased mortality. Since it may much reduce the effects of the host response, the net effect of GALM is probably to reduce mortality $(\alpha<1)$. 
In seeking arbitrary but biologically plausible values for the growth and mortality parameters, to generate the results shown in Figs. 2 to 4 we used a set of 'default' parameters based on Fig. 1. That is, unless otherwise stated, we set the growth rate in host $\mathrm{i}\left(g_{i}\right)$ as half that in host $\mathrm{j}\left(g_{j}\right)$ to reflect the fact that $\mathrm{i}$ may be smaller and may be an ectotherm, and $\mathrm{j}$ is likely to be bigger and may be an endotherm. The constant, sizeindependent mortality rate in $\mathrm{j}\left(p_{j}\right)$ was set equal to the size-independent mortality rate in $\mathrm{i}\left(p_{n i}^{0}\right)$, and the transmission rate from $\mathrm{i}$ to $\mathrm{j}\left(p_{i j}\right)$ was set to twice this value (reflecting predation by a common predator). The constant scaling the magnitude of the sizedependent mortality rate $\left(p_{n i}^{g}\right)$ was set as one tenth that of the size-independent mortality rate $\left(p_{n i}^{0}\right)$, so that mortality rate increases gradually from size at infection $\left(W_{0}\right)$, which was set arbitrarily at a mass of 1.0 unit and the lethal size $\left(W_{m}\right)$ at 10 units.

\subsection{How does GALM affect fitness?}

To explain more fully how the timing of GALM affects fitness, Fig. 2 shows how fitness (proportional to lifetime egg production; for calculation see Appendix A) changes as the size at which GALM occurs $\left(W_{d}\right)$ is varied across its possible range from $W_{0}$ (no growth in host i; i.e. paratenicity) to $W_{m}$ (continuous growth until death of the larva; i.e. no GALM). The lines show fitness at different levels of $\alpha$, from its lowest value at 0 (where GALM removes all size-dependent mortality) through $\alpha$ equals 1 (where mortality after GALM is the same as that immediately before GALM), to high levels where $\alpha$ exceeds 1 , some of which are too high to be biologically plausible, but are shown here for heuristic purposes. As expected, fitness declines with $\alpha$, except in the 
case of accelerating mortality in conditions where the optimum is continuous growth without GALM (Fig. 2b) so that fitness becomes independent of $\alpha$.

In the linear mortality model (Fig. 2a), when GALM removes all size-dependent mortality $(\alpha=0)$, maximum fitness occurs at an intermediate size $\left(W_{d}^{*}=5\right)$. As $p_{n i L}^{(2)}\left(t_{d}\right)$ increases ( $\alpha=0.2, \alpha=0.5$ ), maximum fitness is attained at greater size so that $W_{d}^{*}$ shifts towards $W_{m}$. When $\alpha \approx 0.7$, the best strategy becomes continuous growth until death (no GALM), as can be seen in the line for $\alpha=1$. This strategy is uniquely stable until $\alpha \approx 1.9$ when the fitness plot becomes U-shaped with an intermediate minimum value, so that at $\alpha=2,5$, and 20, the limit strategies (paratenicity or continuous growth, i.e. $\left.W_{0}, W_{m}\right)$ are alternative optima, with the higher peak at $W_{m}$. However, as $\alpha$ increases beyond 64, the local optimum at the lower limit $W_{0}$ is lost, and $W_{m}$ becomes uniquely stable again.

With the same parameter values, the accelerating mortality model behaves differently (Fig. 2b). Because it accelerates to infinity at $W_{m}, p_{n i A}^{(2)}\left(t_{d}\right)$ generates fitness values that are independent of $\alpha$ at the limit $W_{m}$. At $\alpha=0$, there is again an intermediate optimum $\left(W_{d}^{*}=3.8\right)$, but as $p_{n i A}^{(2)}\left(t_{d}\right)$ increases $(\alpha=0.2, \alpha=0.5)$, the fitness maximum moves towards lower size, not higher size as in the linear case (Fig. 2a). At $\alpha \approx 1.9$, paratenicity ( $\left.W_{0}\right)$ becomes the optimal strategy, then bistability occurs around $\alpha \approx 2.2$, initially with GALM at a high intermediate size and with $W_{0}$ the dominant peak, but quickly resolving to bistability at the limits. $W_{m}$ takes over as the dominant peak at $\alpha \approx 2.6$, with the fitness plot becoming increasing U-shaped (see curves for $\alpha=5,20$ ). $W_{m}$ is uniquely stable above $\alpha \approx 65$. 
Although implausibly high $\alpha$ values are used, Fig. 2 demonstrates that, even when all other parameters constant, the effects of the size-dependent larval mortality discontinuity at GALM has complex implications for optimal growth strategies in larval helminths. We next seek qualitative predictions that are independent of the effects of GALM at biologically plausible regions for $\alpha$.

\subsection{How do the growth, transmission and mortality parameters affect optimal size at GALM?}

Figs. 3 and 4 show the effects of varying each growth, transmission or mortality parameter independently from our default values (used in Fig. 2) to examine how each affects growth in relation to the change in mortality rate at GALM, modelled by $\alpha$; they plot the optimal response $W_{d}^{*}(\alpha)$. When there are alternative local optima at a given $\alpha$, Figs. 3 and 4 show the higher of the two optima.

We know of no studies that have examined how helminth mortality rate changes at GALM; this may vary greatly across species, but for small encapsulated or encysted larval helminths our guess is that mortality rate is typically reduced (possibly considerably) at GALM. Thus we anticipate that time-dependent mortality rate changes at GALM are beneficial for the parasite, or only slightly detrimental, and examine in Figs. 3 and 4 the range where $\alpha$ lies between 0 and 1.2. In particular we wish to predict conditions in which the larva should not grow in the intermediate host $\mathrm{i}$, but remain at its size at establishment $\left(W_{d}^{*}=W_{0}\right)$, i.e. where i should be treated as a paratenic host. 
Figs. 3 (for the linear mortality model) and 4 (accelerating mortality model) will be discussed together in relation to each given parameter, which is allowed in each separate graph to vary, while all other parameters are held constant at their default values.

Increasing the cost of each unit of growth in terms of size-dependent mortality parameter, $p_{n i}^{g}$, decreases optimal size at GALM in both models (Figs. 3a, 4a). The linear model appeared more sensitive to changes in $p_{n i}^{g}$, and could readily generate paratenicity or continuous growth at lowest $\alpha$.

The ratio, mortality/growth rates in the next host $\mathrm{j}, p_{j} / g_{j}$, occurs always as a single variable in our analytical solutions. Increasing $p_{j} / g_{j}$ makes $\mathrm{j}$ relatively less attractive for growth and survival and favours increased growth in host i (higher optimal size at GALM) in both models (Figs. 3b, 4b), mirroring the effect of $p_{n i}^{g}$ (Figs. 3a, 4a) but in reverse direction. If $p_{j} / g_{j}$ is low, the parasite economises on growth in i so that it can grow later in $\mathrm{j}$.

The effect of growth rate in host $\mathrm{i}, g_{i}$, differs qualitatively between the two models (Figs. 3c, 4c). If $\alpha<1$, both give the result that the optimal size at GALM decreases with growth rate in host i, $g_{i}$. If $\alpha>1$, the linear model gives $W_{0}$ or $W_{m}$, as predicted for all parameters. However, the accelerating model now has reversed predictions: the optimal size at GALM now increases with growth rate in host $\mathrm{i}, g_{i}$. Increased optimal size at GALM with increased growth rate when $\alpha<1$ is explicable in terms of the benefits of GALM allowing higher growth; whereas if GALM increases mortality $(\alpha>1)$, the reverse applies, so that increasing $g_{i}$ causes $W_{d}^{*}$ to decrease (Fig. 4c), compensating for the higher size-related mortality due to GALM. While the most plausible range is for $\alpha<1$, we cannot dismiss the possibility of a reversed effect of $g_{i}$ when $\alpha>1$. 
The parameters $p_{n i}^{0}$ (the size or time-independent mortality rate) and $p_{i j}$ (the transmission rate to host $\mathrm{j})$ always act as a single variable $\left(\gamma=p_{n i}^{0}+p_{i j}\right)$ in the solution for optimal size at GALM. They have equivalent qualitative effects: optimal growth increases with $\gamma$ (Figs. 3d, 4d). Increasing $p_{n i}^{0}$ and $p_{i j}$ decrease the expected time spent in host $\mathrm{i}$, and unlike $p_{n i}^{g}$, they do so independent of size or time. A shorter expected time in i allows greater growth at no extra cost, hence optimal size increases with $\gamma$. At the limit $\alpha=0$ we obtain for linear mortality

$$
W_{d}^{*}=p_{j} g_{i} / p_{n i}^{g} g_{j}
$$

and for accelerating mortality

$W_{d}^{*}=W_{m}-\left(W_{m}-W_{0}\right)^{2} /\left(p_{j} g_{i} / p_{n i}^{g} g_{j}+W_{m}-2 W_{0}\right)$.

Thus if GALM offers a perfect safe haven from time-dependent mortality, random mortality rates and transmission rates $\left(p_{n i}^{0}, p_{i j}\right)$ do not affect growth strategy, $W_{d}^{*}$ (see Figs. 3d, $4 \mathrm{~d}$ at $\alpha=0$ ).

In summary, while the linear model cannot yield intermediate growth for $\alpha>1$ (it generates either paratenicity or continuous growth), the more biologically realistic accelerating model can readily give intermediate solutions in this region. The difference between the two models for variations in most parameters is qualitative rather than quantitative: optimal size increases with $p_{n i}^{g}$ and $\gamma=p_{n i}^{0}+p_{i j}$, and decreases with $p_{j} / g_{j}$. For a given set of parameters, the relation between optimal size at GALM, $W_{d}^{*}$, and $\alpha$ may be either increasing or decreasing between $0<\alpha<1$, depending on the values of the parameters. Both models predict increased size with increased growth rate, $g_{i}$ up to $\alpha=1$, but the predictions of the accelerating model reverse when $\alpha>1$. 


\subsection{The effects of parasite intensity on growth of each larva}

The effect of competition on optimal larval growth strategy is a complex problem requiring further study (see Parker et al. $2003 \mathrm{~b}$ for a simple approach). The most direct assumption is that size-dependent mortality relates only to the total mass of all competing larvae independent of their number, so that one larva of size 10 units experiences the same size-dependent death rate as each of 10 larvae of size 1 unit. Assuming also that $N$ larvae of the same species enter host i simultaneously, that $\alpha=1$, and that larvae can vary only $W_{d}$, pilot work using the present approach (see BPC) showed that the optimal solution is for total larval mass to remain constant with $N W_{d N}^{*}=W_{d}^{*}$, where $W_{d N}^{*}$ is the optimal size of each competing larva at GALM. Although more comprehensive analyses are planned, this simple result may hold to a first approximation. Thus, under the assumption that size-dependent mortality is equivalent to the total mass of larvae, then (very approximately) our solutions for $W_{d}^{*}$ can be seen as the total parasite mass. The work of Parker et al. (2003b) suggested that $N W_{d}^{*}>N W_{d N}^{*}>W_{d}^{*}$, so that both results indicate that optimal size at GALM should reduce with parasite intensity.

However, such assumptions may not be universally valid. Where time-dependent mortality of the larva relates to its host's response, the assumption that size-dependent death rate relates only to total parasite mass is unlikely to apply. While there may well be an increase in mortality with total parasite mass, mortality is unlikely to increase in direct proportion to total mass, since the mortality increase will be more dependent on time than 
on size. In the extreme case where only time (but not parasite mass) is important, then our analysis would be independent of intensity, with $W_{d N}^{*}=W_{d}^{*}$.

\subsection{Summary of results}

We can see that paratenicity, or at least very low growth, in host $\mathrm{i}$ is most likely with high values for:

(i) $W_{0}$, the larval size at establishment in i,

(ii) $p_{n i}^{g}$, the constant that scales the size- or time-dependent mortality rate in i,

(iii) $\mathrm{N}$, the intensity of parasite larvae in host $\mathrm{i}$, and with low values for

(iv) $p_{n i}^{0}$, the random or time-independent mortality rate,

(v) $p_{i j}$, the transmission rate of the larva between host $\mathrm{i}$ and host $\mathrm{j}$,

(vi) $p_{j} / g_{j}$, the ratio of noise mortality rate relative to growth rate in host $\mathrm{j}$,

(vii) $g_{i}$, the growth rate in i, provided that GALM reduces the mortality rate in host $\mathrm{i}$.

Obviously the opposite conditions favour growth in host $i$, so that host $i$ is treated as an intermediate host.

\subsection{Generality of results}

Our analysis assumes specific functions (linear and hyperbolic) for the timedependent mortality rates before and after GALM; the hyperbolic function is the more plausible biologically. As with all such investigations, the question arises whether the 
form of these functions influences the results qualitatively. There are clearly many possible ways that these noise mortality functions might change with time, including the discontinuity at GALM. For example, $\alpha$ might change with $t_{d}$, which would generate further complexity.

We have investigated other forms, including (in detail) the accelerating form suggested by BPC, in which

$$
p_{n i}^{(1)}(t)=p_{n i}^{0}+p_{n i}^{g} /\left(W_{m}-W_{0}-g_{i} t\right) .
$$

At establishment in host i $(t=0)$, this has a much lower gradient $\left(d p_{n i}^{(2)} / d t_{d}=p_{n i}^{g} g_{i} /\left[\left(W_{m}-W_{0}\right)^{2}\right]\right)$ and size-dependent component $\left(p_{n i}^{g} /\left(W_{m}-W_{0}\right)\right)$ than Eq. (4). The equation equivalent to Eq. (6) is

$$
W_{d}^{*}=W_{m}-p_{n i}^{g} / E \text {. }
$$

Using

$$
p_{n i}^{(2)}\left(t_{d}\right)=p_{n i}^{0}+\alpha p_{n i}^{g} /\left(W_{m}-W_{0}-g_{i} t_{d}\right),
$$

this model can also give rise to all three possible growth strategies and yields the same qualitative conclusions as in Section 3.4, but generates quantitatively different results from either Figs. 3 or 4 in its response to $\alpha$.

Our models suggest that the parasite's strategic response associated with the discontinuity at GALM will be complex, and may vary considerably depending on the explicit forms of $p_{n i}^{(1)}(t)$ and $p_{n i}^{(2)}\left(t_{d}\right)$. We nevertheless anticipate that the conclusions outlined in Section 3.4 will hold generally for most simple monotonic increasing forms, though this remains to be confirmed. 


\section{Discussion}

In summary, we have modelled optimal growth strategies of trophically transmitted helminth larvae in their intermediate hosts. We propose that the noise mortality rate of a larva varies with time $t$ post infection (Fig. 1), and consists of two discontinuous components: (i) before GALM occurs at time $t_{d}$, noise mortality rate increases as $p_{n i}^{(1)}(t)$, and (ii) after GALM, it remains constant with time at level $p_{n i}^{(2)}(t)=p_{n i}^{(2)}\left(t_{d}\right)$. This constant level of noise mortality after GALM, $p_{n i}^{(2)}\left(t_{d}\right)$, increases monotonically with the time at which GALM occurs, $t_{d}$ (Fig. 1).

The three possible larval growth strategies in the intermediate host are: (i) no growth (immediate GALM; paratenicity), (ii) growth to an intermediate optimum, ending with GALM, or (iii) continuous growth until the larva dies (no GALM). Both linear (Fig. 1a, b) and accelerating (Fig. 1c, d) mortality models analysed here can generate all three possibilities for optimal growth in host $\mathrm{i}$, depending on the various mortality and growth parameters and on the nature of the discontinuity at GALM, modelled by factor $\alpha$ (see Figs. 2, 3 and 4). Examples of all three strategies occur in the life cycles of parasitic helminths. Though not as ubiquitous as growth to GALM, paratenicity is nevertheless common in some helminth groups, especially in second hosts in three host life cycles (see, for example, Anderson, 2000 for nematodes of vertebrates; Schmidt, 1985 for acanthocephalans). Growth up to some form of GALM is the most typical strategy in all groups, and dominant in cestodes and trematodes. The strategy for continuous parasite larval growth without GALM occurs, for example, in Diphyllobothrium dendriticum plerocercoids in brown trout (second intermediate host, Rahkonen et al., 1996) and also 
Taenia taeniaeformis strobilocerci in lambs (first intermediate host, Sweatman \& Plummer, 1957).

Our results can be categorised into: (i) effects that depend on the mortality discontinuity at GALM, i.e. direct qualitative effects of $\alpha$, or parameter effects that change sign with $\alpha$, and (ii) parameter effects that are independent of GALM, i.e. provided that an intermediate growth solution is possible, increasing the parameter always either increases or decreases larval growth, whatever the value of $\alpha$. We first discuss biological aspects of the discontinuity at GALM (Section 4.1), followed by circumstantial evidence for growth predictions that are qualitatively independent of the discontinuity at GALM (Section 4.2). We then discuss briefly some effects of host parasite coevolution (Section 4.3), and finally stress the importance of paratenicity as a growth strategy in the parasitological context (Section 4.4).

\subsection{Effects of the discontinuous change in mortality rate at GALM}

After migration and growth in the intermediate host's tissues, helminth larvae may undergo various changes associated with GALM. A larva may (i) remain free within the host tissues, or (ii) secrete a cyst around itself (encystment), or (iii) the host may lay down new tissues to isolate it (encapsulation), or (iv) both encystment with encapsulation may occur (Erasmus, 1972; Olsen, 1974). Both encystment, and encystment with encapsulation, are common in trematodes (see Kearn, 1998); encapsulation occurs widely in nematodes (Anderson, 2000), though not encystment. These changes, coupled with the 
cessation of growth and movement through the tissues, are likely to have significant effects on larval mortality rate.

\subsubsection{GALM: escape to safety, or flight into danger?}

A major influence on our predictions is the effect of GALM on the size-dependent mortality rate, modelled by the factor $\alpha$ (below, Section 4.1.2). Unfortunately, we know of no published measurements of changes in parasite death rate at GALM. Intuition suggests that for helminth larvae of vertebrates, GALM and any associated encapsulation or encystment typically act to reduce noise mortality, i.e. $0<\alpha<1$. Both encapsulation and encystment may effectively isolate the larva, reducing parasite mortality by averting host immune responses. Rapid isolation and GALM may facilitate long-term survival in the host, at least initially providing a safe haven for the larva. In fish, amphibian and reptile paratenic hosts, encapsulated larvae may survive quiescently for very long durations. For example, renicolid trematodes commonly survive after GALM as encysted larvae throughout the lifetime of their herring host, often years (e.g. Campbell et al., 2007), possibly supporting the reduced mortality $(0<\alpha<1)$ hypothesis. Trematode encystment in invertebrates may also reduce noise mortality after GALM by protecting larvae from host responses.

Invertebrate host responses are very different from those of vertebrates (Beck and Habicht, 1996). There is usually little apparent host response against helminths in their typical (specific) invertebrate hosts. In atypical hosts, larval death commonly follows 
encapsulation and similar responses. However, whether encapsulation in invertebrate hosts increases mortality when (rarely) it occurs in specific hosts is unclear.

In specific hosts, encystment and encapsulation presumably constrain space and resources for growth, limiting further growth in what may represent an immunologically safe haven. This is presumably why encystment/encapsulation are generally accompanied by GALM. Constraints to growth may also occur when parasites avoid host immune responses in other ways. For example, the Trichinella L1 stage larva develops inside a muscle cell ('nurse cell', Anderson, 2000). This is immunologically inert for the host, so the parasite is protected though its growth is restricted. Some nematode infective stages (L3) retain the skin of the L2 stage (Anderson 2000), possibly for protection against host responses, but which also constrains growth. Cercariae of the trematode Diplostomum pseudobaeri infect fish second intermediate hosts, migrate to, grow and undergo GALM in the host's retina, (which is immunologically inert; Field \& Irwin, 1995), where they can survive for at least four years (Shigin, 1964). Why growth stops is unclear, unless too much growth would decrease survival of the host, and hence parasite.

Thus from current evidence, size-dependent mortality most plausibly typically decreases at GALM $(0<\alpha<1)$, but we cannot preclude the possibility that it increases ( $\alpha$ $>1$ ) in some circumstances. For instance, encystment must incur some small energy cost to the larva that may subsequent increase mortality. We have shown that GALM can be beneficial even when it increases mortality $(\alpha>1)$, by precluding even more damaging death rates due eventually to continued growth. A human analogy might be that chemotherapy, while harmful in the short term, arrests growth of cancers that will ultimately become much more harmful. 


\subsubsection{Effects of GALM on growth predictions}

The effects of the various parameters in relation to $\alpha$ have been discussed in Section 3.2. To summarise: if growth costs $\left(p_{n i}^{g}\right)$ are unfavourably high (Figs. 3a, 4a), or the mortality/growth rate ratio in $\mathrm{j}\left(p_{j} / g_{j}\right)$ favourably low (Figs. $\left.3 \mathrm{~b}, 4 \mathrm{~b}\right)$, a safe haven effect typically promotes paratenicity (the safe haven of GALM outweighs the benefits of highrisk growth). The effects on growth of time-independent mortality and transmission rate (the combined parameter $\gamma=p_{n i}^{0}+p_{i j}$ ) are independent of $\gamma$ at $\alpha \rightarrow 0$, but become strongly dependent on $\gamma$ as $\alpha$ increases (Figs. 3d, $4 \mathrm{~d})$. With each parameter $\left(p_{n i}^{g}, p_{j} / g_{j}\right.$, $\gamma)$, as $\alpha$ increases, optimal size at GALM, $W_{d}^{*}(\alpha)$, may increase or decrease across the most plausible biological range $(0<\alpha<1)$, but their qualitative effects on growth are independent of $\alpha$. If $W_{d}^{*}(\alpha)$ is decreasing, further increases in $\alpha$ typically result in paratenicity before ultimately (often at implausibly high $\alpha$ not shown in Figs. 3 and 4), the optimal strategy becomes continuous growth to death. The growth rate in host i, $g_{i}$, is the only parameter having an effect that in the accelerating model changes qualitatively as $\alpha$ increases (Fig. 4c); the same effect was apparent with accelerating form proposed in BPC.

\subsection{Evidence for the predictions - what causes paratenicity?}

The occurrence of paratenicity in helminths with complex life cycles is quite variable. In trematodes, paratenic hosts occur rarely (e.g. Azygia lucii, Ginetsinskaya, 
1958; Genarchopsis goppo, Madhavi, 1978). In cestodes, where there is a second intermediate host, growth typically continues until GALM, or more rarely, until death (see Section 4.1.2). Paratenic tendencies are sometimes shown when a larva from a smaller fish is ingested by a larger one (e.g. transfer of Diphyllobothrum latum plerocercoids from perch to pike; von Bonsdorff, 1977). In nematodes and acanthocephalans, paratenicity is much more frequent (preliminary investigations suggest 10 to 20 percent of species, depending on the group).

Predictions for growth, mortality and transmission rates on paratenicity and are listed in Section 3.4. Direct biological support is currently unavailable, however, in the following Sections we offer some speculative evidence.

\subsubsection{Effects of parasite mass relative to host mass; high costs of growth}

The maximum size, $W_{m}$, that a larva can attain, and the size at infection, $W_{0}$, must be considered relative to host size; the absolute value of $W_{m}$ will increase with host size. Where time-dependent mortality relates to parasite mass harming its host (rather than host responses), paratenicity is more likely if the infective larva is relatively large.

In acanthocephalans and nematodes, paratenicity typically occurs in a second host after active larval growth in a first (intermediate) host. If they infect the second host at high intensities at establishment such larvae may already be of a size where further parasite mass might cause harm ( $W_{0}$ relatively high). Paratenic second hosts in threehost life cycles often accumulate high intensities of helminths (e.g. up to 1000 
acanthocephalans in one Antaractic marine fish; Zdzitowiecki, 1986), increasing the probability of paratenicity.

In trematodes, where asexual reproduction is typical in the first (mollusc) intermediate host (Type II cycle, sensu Parker et al., 2003a), there are virtually no cases of paratenicity in the next host. Because asexual reproduction effectively reduces the mass of each propagule produced in the mollusc (roughly by factor $1 / n$, where $n$ is the number of propagules), size at establishment, $W_{0}$, in the next host may be smaller relative to host size than in helminths lacking asexual reproduction (Type I cycles).

The optimal size at GALM $\left(W_{d}^{*}\right)$ should be greater in larger hosts, since maximum size $\left(W_{m}\right)$ is larger. The size at which parasite growth stops has sometimes been positively related to host size. In some adult cestodes, larger size at maturity occurs in larger definitive hosts (Molnár, 1968, 1977). Similar effects for asymptotic larval size are less known, but do occur (e.g. Schistocephalus solidus procercoids in copepods; Wedekind et al., 2000).

\subsubsection{Low transmission rates from i to $j$, and low time-independent mortality in $i$}

There is circumstantial evidence that low transmission rate $\mathrm{i}$ to $\mathrm{j}\left(p_{i j}\right)$ is associated with paratenicity. Olsen (1974) saw paratenicity in trematodes as "an adaptation by the parasite to survive due to a shortage of definitive hosts...", which is equivalent to low $p_{i j}$. Many larval parasites have long-term residence in paratenic hosts (Esch \& Fernández, 1993), suggesting low transmission rates $\left(p_{i j}\right)$ and low random mortality $\left(p_{n i}^{0}\right)$. 
Parasites that increase $p_{i j}$ by host manipulation should show less paratenicity than comparable parasites lacking manipulation. In parasites with paratenic hosts, a literature survey suggests that manipulation has not been reported at that stage in the life cycle. Moore (2002) states “...paratenic hosts are probably underreported in the literature because there is often little to say about their discovery other than for the record."

Paratenicity arising through low transmission or low random mortality rates allows more time for movement into new habitats and exposure to new potential hosts, possibly increasing the probability of their incorporation.

However, because host i will often be low down the food chain, $p_{i j}$ and $p_{n i}^{0}$ may typically be relatively high, especially since $\mathrm{j}$ is likely to be the most common predator of i. The time that the parasite is likely to reside in host $i$ is thus often short, reducing the relative costs of growth and offering a general reason why paratenicity may be less common than growth in intermediate hosts.

\subsubsection{High time- and/or size-dependent noise mortality through host immune responses}

Parasite growth may be limited by the host's immune response, which takes time to develop. For example, host immunity constrains adult size and fecundity of the nematode Strongyloides ratti (e.g. Viney et al., 2006; Wilkes et al., 2004). Immune responses may also increase with parasite mass (Catadori et al., 2005; Shudo \& Iwasa, 2001) suggesting that immunity generates both time- and size-increasing noise mortality.

\subsubsection{Intensity - high numbers of larvae per host $i$}


High larval intensities may generate paratenicity. Whether individual larvae show paratenicity in response to high intensity will depend on information about competitors. If other larvae cannot be detected, a high mean intensity may select for obligate paratenicity. If larvae can detect others, facultative paratenicity may be optimal at high intensity, and growth at low intensity.

'Crowding effects', decreasing relationships between parasite size and number (Read, 1951), have been widely investigated in adult helminths (see Bush and Lotz, 2000). Such studies are less frequent for larval parasites. In both intermediate hosts of the cestode Schistocephalus solidus (copepods, Michaud et al., 2006; sticklebacks, Heins et al., 2002), the ratio of parasite to host mass becomes high. If larvae can detect competitors, then as expected, early-established larvae grow, but those establishing later do not. In concurrent high density infections, asymptotic size is reduced for all larvae. In successive infections, later-established larvae show reduced growth, and asymptotic size, if reached, is reduced, or the larvae hardly grow at all. Many interpretations are possible for this phenomenon (see Michaud et al., 2006, Heins et al., 2002) including those from our model.

The 'peak shift' phenomenon in adult helminths (e.g. Catadori et al., 2005) suggests that parasite mortality through host immune responses increases with the integral of parasite mass through time. This may also apply for larvae, favouring facultative paratenicity for later larvae.

\subsubsection{Low growth rate in host $i$}


Low growth rate in $\mathrm{i}, g_{i}$, means that longer time and hence higher mortality must be sustained to achieve a given size. For the most plausible values for $\alpha(0<\alpha<1)$ we predict greater size at GALM as $g_{i}$ increases.

Because host $\mathrm{i}$ is lower down the food chain than $\mathrm{j}$, we expect typically lower growth rates in $\mathrm{i}$ than $\mathrm{j}$ because of host i's smaller size (e.g. Clarke, 1954). Also, where (commonly) transmission is from an ectotherm $i$ to an endotherm $j$, this growth rate difference is likely to be much amplified. Our models predict that a huge growth rate in $\mathrm{j}$ relative to i will promote paratenicity or very limited growth in i - growth is deferred until the parasite reaches $\mathrm{j}$.

A problem with testing predictions about paratenicity in relation to growth rate in i is that in paratenic hosts, there is no growth; the potential growth rate, $g_{i}$, cannot be measured.

\subsubsection{Low mortality rate/growth rate in host $j$}

Despite several reasons for expecting low mortality rate/growth rate ratios for host $\mathrm{j}$ (high trophic level, large body size, likely to be endothermic; see also Parker et al., 2003a), establishment in $\mathrm{j}$ does not necessarily guarantee low mortality. In fishes, Hopkins (1959) observed only 1 percent of the cestode Proteocephalus filicollis in three spined sticklebacks surviving to release eggs, whilst Chubb (1963) found a dynamic equilibrium with Triaenophorus nodulosus in pike intestines, generating a more or less constant intensity in fish of a given length throughout the year. 
In Acanthocephala, paratenic hosts commonly occur before the definitive host if this is a bird or mammal (i.e. an endotherm), but less frequently if the definitive host is another ectotherm, e.g. a fish (see data in Schmidt, 1985). Endothermic predators offer much higher growth rates and reduced mortality, explaining why the fish host is treated as paratenic if the definitive host is a mammal or bird (very low $p_{j} / g_{j}$ and $\beta \equiv p_{j} g_{i} / g_{j}$ ).

\subsection{Host-parasite coevolution and the likelihood of paratenicity}

BPC summarised effects of coevolutionary changes in Eq. (1) parameters due to host-parasite conflict. Defence by host i may increase $p_{n i}^{g}$, which scales the mortality rate increase during parasite growth, increasing the probability of paratenicity. Evolved host manipulation may reduce the probability of paratenicity by increasing the transmission rate, $p_{i j}$.

Of key interest will be evolutionary changes in mortality rate after GALM affecting $p_{n i}^{(2)}$, or specifically, $\alpha$, which greatly influences the strategic option between growth and paratenicity. The evolution of encystment, a parasite response, is likely to reduce mortality at GALM. Encapsulation, a host response, may initially have increased parasite mortality, but its deleterious effects have been shown empirically to reduce over evolutionary time (Kraaijeveld et al., 2001). Further, if encapsulation is a host response that reduces parasite harm to the host by isolating it from host tissues (see Section 4.1.1), $\alpha$ is again likely to reduce through selection on parasite resistance; parasites have evolved high resistance to their specific hosts' immune responses. Thus when a parasite larva stops moving through the host tissues, undergoes GALM, and allows encapsulation, 
this is probably an evolved parasite strategy which reduces its mortality rate (as in the cestode Triaenophorus nodulosus in perch livers, see Chubb, 1964; Kuperman, 1981).

\subsection{In conclusion: paratenicity as a growth strategy}

Paratenic hosts can be facultative, often occurring when an intermediate host $\mathrm{i}$ is not eaten by the definitive host $\mathrm{j}$, but by an alternative predator which then becomes a paratenic host (e.g. Bothriocephalus gregarius, Morand et al., 1995). Such transmission events are probably less common than the shorter more typical cycle. This 'less common' host effect may constitute one major opportunity for the evolution of paratenicity, relating to the costs of increasing the host spectrum, i.e. to the costs of generalism (Parker et al., 2003a; BPC). Helminths have high resistance to their usual hosts $(\mathrm{i}, \mathrm{j})$, but much less resistance to hosts that are less commonly utilised (k). Host $\mathrm{k}$ may be a lateral host to i. An explanation relates to the costs of additional resistance mechanisms when the transmission pathway $p_{i k}$ is less frequent than $p_{i j}$. If the parasite remains poorly adapted to host $\mathrm{k}$ for adaptive economic reasons, its mortality rate, were it to grow actively in $\mathrm{k}$, would be high. Thus if GALM represents a safe haven, allowing an immediately reduction in mortality, paratenicity is predicted to be a common adaptive strategy for such hosts. This 'less common host' effect cannot, however, explain obligate paratenicity at a given stage in a cycle (see Anderson, 2000 for nematodes; Schmidt, 1985 for acanthocephalans).

Parasite larvae show great variation in growth strategies in their hosts: here we have attempted to create a predictive framework for this diversity. In particular, we urge that paratenicity should be recognised as one component of a set of growth strategies, rather 
than as a 'transport' event or an 'ecological bridge' (e.g. Poinar et al., 1976; Marcogliese, 2001) in a complex helminth life cycle, which is equally applicable to intermediate hosts. As stressed in the Introduction, the fundamental distinction between intermediate and paratenic hosts relates to the growth and life history strategy of the helminth larva. There would be much to commend a change in terminology from 'intermediate host' and 'paratenic host', to the single term 'intermediate host' for any host preceding a definite host in a life cycle. Intermediate hosts could then be termed either 'growth intermediate hosts' or 'paratenic intermediate hosts' to reflect accurately the parasite's growth response in any host prior to the definitive host in a life cycle.

\section{Acknowledgements}

We are indebted to the Natural Environment Research Council for supporting part of this work (Grant GR3/12774), and are most grateful to a very perceptive anonymous reviewer.

\section{Appendix A: Iteration of fitness and other tests of the solution for optimal size at}

\section{GALM}

BPC (Ball et al., 2008.) showed that expected number of eggs produced by the parasite in host $\mathrm{j}$ (our fitness measure) is

$$
E\left(t_{d}\right)=\left(w_{i j 1}+w_{i j 2}\right)\left(1-\phi_{d}\right),
$$


where $w_{i j 1}$ is the number of eggs produced by a mutant that transfers to $\mathrm{j}$ before it reaches size $W_{d}, w_{i j 2}$ the number produced by a mutant that transfers to $\mathrm{j}$ after it reaches size $W_{d}$, and $\left(1-\phi_{d}\right)$ is a constant relating to the costs of generalism (see BPC).

To examine the relation between fitness and $t_{d}$, we iterated solutions for fitness at given values of $a$, from the following equations (BPC):

$$
\begin{aligned}
& w_{i j 1}=A \int_{0}^{t^{d}} e^{p_{j} g_{i} t / g_{j}} n_{i}\left(t, t_{d}, g_{i}\right) d t \\
& w_{i j 2}=A e^{p_{j} g_{i} t_{d} / g_{j}} n_{i}\left(t_{d}, t_{d}, g_{i}\right) / p_{i}^{d}\left(t_{d}\right)
\end{aligned}
$$

where $A=\left(b_{j} W_{j}^{*} / p_{j}\right) P_{e j} p_{i j} e^{-p_{j}\left(W_{j}^{*}-W_{0}\right) / g_{j}}$ (which contains only constants related to parameters in host $\mathrm{j}$, see BPC Eq. (10) ), and $p_{i}^{d}\left(t_{d}\right)=\left(p_{n i}^{(2)}\left(t_{d}\right)+p_{i j}\right)$. While (A.2) can be integrated, (A.1) cannot, and was iterated numerically using the software package proFit.

For the linear mortality case (BPC; Eq. (A.8)),

$$
\begin{aligned}
& n_{i}(t)=P_{i} e^{-\left(\left(p_{n i}^{0}+p_{n i}^{g} W_{0}+p_{i j}\right) t+p_{n i}^{g} g_{i} t^{2} / 2\right)} \text { for } t \leq\left(W_{m}-W_{0}\right) / g_{i} ; \\
& n_{i}(t)=0 \text { otherwise. }
\end{aligned}
$$

BPC used a simpler version of the accelerating mortality case (their Eq. (7))

$$
p_{n i}^{(1)}(t)=p_{n i}^{0}+p_{n i}^{g} /\left(W_{m}-W_{0}-g_{i} t\right),
$$

which (after correcting typographical errors in BPC Eq. (A.9)) gave

$n_{i}(t)=P_{i} e^{-\left(\left(p_{n i}^{0}+p_{i j}\right) t\right)}\left[\left(W_{m}-W\right) /\left(W_{m}-W_{0}\right)\right]^{p_{m}^{\prime} / g_{i}}$ for $t \leq\left(W_{m}-W_{0}\right) / g_{i} ;$

$n_{i}(t)=0$ otherwise.

For the accelerating mortality case used in the present paper (Eq. (4)), we obtained:

$n_{i}(t)=P_{i} e^{-\left(p_{n i}^{0}+p_{n i}^{g}\left(2 W_{0}-W_{m}\right)+p_{i j}\right) t}\left[\left(W_{m}-W\right) /\left(W_{m}-W_{0}\right)\right]^{p_{i i}^{g}\left(W_{m}-W_{0}\right)^{2} / g_{i}}$ for $t \leq\left(W_{m}-W_{0}\right) / g_{i} ;$ $n_{i}(t)=0$ otherwise. 
We also checked for paratenic solutions that the gradient $d E\left(t_{d}=0\right) / d t_{d}<0$, i.e. that a local optimum occurs at $t_{d}=0$, from Eq. (11). We confirmed analytical solutions in regions where size at GALM is predicted analytically to be intermediate $\left(W_{0}<W_{d}^{*}<W_{m}\right)$ by iterations of fitness. Where optima exist at the end points $\left(W_{0}, W_{m}\right)$ the quadratic solutions could not be used and such regions were also determined by fitness iterations.

\section{REFERENCES}

Anderson, R.C., 2000. Nematode Parasites of Vertebrates their Development and Transmission. Second Edition. CABI Publishing, Wallingford.

Ball, M.A., Parker, G.A., Chubb, J.C., 2008. The evolution of complex life cycles when parasite mortality is size- or time-dependent. J. Theor. Biol. 253, 202-214.

Beck, G., Habicht, G.S., 1996. Immunity and the invertebrates. Sci. Am. 275, 60-66.

Bonsdorff, B. von, 1977. Diphyllobothriasis in Man. Academic Press, London.

Bush, A.O., Lotz, J.M., 2000. The ecology of “crowding”'. J. Parasit. 86, 212-213.

Bush, A.O., Fernández, J.C., Esch, G.W., Seed, R.J., 2001. Parasitism: the Diversity and Ecology of Animal Parasites. Cambridge University Press, Cambridge.

Campbell, N., Cross, M.A., Chubb, J.C., Cunningham, C.O., Hatfield, E.M.C., MacKenzie, K., 2007. Spatial and temporal variations in parasite prevalence and infracommunity structure in herring (Clupea harengus L.) caught to the west of the British Isles and in the North and Baltic Seas: implications for fisheries science. J. Helminth. 81, 137-146.

Catadori, I.M., Boag, B., Bjørnstad, O.M., Cornell, S.J., Hudson, P.J., 2005. Peak shift and epidemiology in a seasonal host-nematode system. Proc. R. Soc. B 272, 1163-69. 
Chubb, J.C., 1963. Seasonal occurrence and maturation of Triaenophorus nodulosus (Pallas, 1781)(Cestoda: Pseudophyllidea) in the pike Esox lucius L. of Llyn Tegid. Parasitology 53, 419-433.

Chubb, J.C., 1964. Observations on the occurrence of the plerocercoids of Triaenophorus nodulosus (Pallas, 1781)(Cestoda: Pseudophyllidea) in the perch Perca fluviatilis L. of Llyn Tegid (Bala Lake), Merionethshire. Parasitology 54, 481-491.

Clarke, A.S., 1954. Studies on the life cycle of the pseudophyllidean cestode Schistocephalus solidus. Proc. Zool. Soc. Lond. 124, 257-304.

Erasmus, D.A., 1972. The Biology of Trematodes. Edward Arnold, London.

Esch, G.W., Fernández, J.C., 1993. A Functional Biology of Parasitism. Chapman \& Hall, London.

Field, J.S., Irwin, S.W.B., 1995. Life-cycle description and comparison of Diplostomum spathaceum (Rudolphi, 1819) and D. pseudobaeri (Razmaskin \& Andrejak, 1978) from rainbow trout (Oncorhynchus mykiss Walbaum) maintained in identical hosts. Parasit. Res. 81, 505-517.

Ginetsinskaya, T.A., 1958. The life cycles of fish helminths and the biology of their larval stages. In: Dogiel, V.A., Petruschevski, G.K., Polyanski, Yu.I. (Eds.), Parasitology of Fishes. (English translation by Z. Kabata). Oliver \& Boyd, London, pp. 140-179.

Heins, D.C., Baker, J.A., Martin, H.C., 2002. The “crowding effect"' in the cestode Schistocephalus solidus: density-dependent effects on plerocercoid size and infectivity. J. Parasit. 88, 302-307. 
Hopkins, C.A., 1959. Seasonal variations in the incidence and development of the cestode Proteocephalus filicollis (Rud. 1810) in Gasterosteus aculeatus (L. 1776). Parasitology 49, 529-542.

Iwasa, Y., Wada, G., 2006. Complex life cycle and body sizes at life-history transitions for macroparasites. Evol. Ecol. Res. 8, 1427-1443.

Kearn, G.C., 1998. Parasitism and the Platyhelminthes. Chapman \& Hall, London.

Kraaijeveld, A.R., Hutcheson, K.A., Limentani, E.C., Godfray, H.C.J., 2001. Costs of counter defenses to host resistance in a parasitoid of Drosophila. Evolution 55, 1815-1821.

Kuperman, B., 1981. Tapeworms of the Genus Triaenophorus, Parasites of Fishes. (English translation). Amerind, New Delhi.

Madhavi, R., 1978. Life history of Genarchopsis goppo Ozaki, 1925 (Trematoda: Hemiuridae) from the freshwater fish Channa punctata. J. Helminth. 52, 251-259. Marcogliese, D.J., 2001. Pursuing parasites up the food chain: implications of food web structure and function on parasite communities in aquatic systems. Acta Parasit. 46, 82-93.

Michaud, M., Milinski, M., Parker, G.A., Chubb, J.C., 2006. Competitive growth strategies in intermediate hosts: experimental tests of a parasite life-history model using the cestode, Schistocephalus solidus. Evol. Ecol. 20, 39-57.

Molnár, K., 1968. Bothriocephalus phoxini sp. n. (Cestoda, Pseudophyllidea) from Phoxinus phoxinus L. Folia Parasit. Praha 15, 83-86.

Molnár, K., 1977. On the synonyms of Bothriocephalus acheilognathi Yamaguti, 1936. Parasit. Hung. 10, 61-62. 
Moore J., 2002. Parasites and the Behaviour of Animals. Oxford University Press, Oxford.

Morand, S., Robert, F., Connors, V.A., 1995. Complexity in parasite life cycle: population biology of cestodes in fish. J. Anim. Ecol. 64, 256-264.

Olsen, O.W., 1974. Animal Parasites: their Life Cycles and Ecology. University Park Press, Baltimore.

Parker, G.A., Chubb, J.C., Ball, M.A., Roberts, G.N., 2003a.. Evolution of complex life cycles in helminth parasites. Nature, London 425, 480-484.

Parker, G.A., Chubb, J.C., Roberts, G.N., Michaud, M., Milinski, M., 2003b. Optimal growth strategies of larval helminths in their intermediate hosts. J. Evol. Biol. 16, 4754.

Poinar, G.O., Jr., Lane, R.S., \& Thomas, G.M., 1976. Biology and redescription of Pheromermis pachysoma (v. Linstow) n. gen., n. comb. (Nematoda: Mermithidae), a parasite of yellowjackets (Hymenoptera: Vespidae). Nematologia 22, 360-370.

Rahkonen, R., Aalto, J., Koski, P., J. Särkkä, Juntunen, K., 1996. Cestode larvae Diphyllobothrium dendriticum as a cause of heart disease leading to mortality in hatchery-reared sea trout and brown trout. Dis. Aquat. Org. 25, 15-22.

Read, C.P., 1951. The "crowding effect' in tapeworm infections. J. Parasit. 37, 174178.

Scharsack, J.P., Koch, K., Hammerschmidt, K., 2007. Who is in control of the stickleback immune system: interactions between Schistocephalus solidus and its specific vertebrate host. Proc. R. Soc. Lond. B. 274, 3151-3158. 
Schmidt, G.D., 1985. 8 Development and life cycles. In: Biology of the Acanthocephala Crompton, D.W.T., Nickol, B.B. (Eds.) Cambridge University Press, Cambridge, pp. 273-305.

Shigin, A.A., 1964. The life span of Diplostomum spathaceum in the intermediate host. Trudy Gelmint. Lab. 14, 262-272.

Shudo, E., Iwasa, Y., 2001. Inducible defense against pathogens and parasites: optimal choice among multiple options. J. Theor. Biol. 209, 233-247.

Sweatman, G.K., Plummer, P.J.G., 1957. The biology and pathology of the tapeworm, Taenia hydatigena in domestic and wild hosts. Can. J. Zool. 35, 93-109.

Viney, M.E., Steer, M.D., Wilkes, C.P., 2006. The reversibility of constraints on size and fecundity in the parasitic nematode Strongyloides ratti. Parasitology 133, 477-483.

Wedekind, C., Christen, M., Schärer, L., Treichel, N., 2000. Relative helminth size in crustacean hosts: in vivo determination, and effects of host gender and within-host competition in a copepod infected by a cestode. Aq. Ecol. 34, 279-285.

Wilkes, C.P., Thompson, F.J., Gardner, M.P., Paterson, S., Viney, M.E., 2004. The effect of the host immune response on the parasitic nematode Strongyloides ratti. Parasitology 128, 661-669.

Zdzitowiecki, K., 1991. Antarctic Acanthocephala. Synopses of the Antarctic Benthos Volume 3. Wägele, J.W., Sieg J., (Eds.). In: Fricke, R., (Ed.), Theses Zoologicae, Volume 15, pp. 1-115. 


\section{Legends to the Figures}

Fig. 1. Noise mortality rate, $p_{n i}$, in relation to time $t$ spent in the host. The continuous line shows the relation between mortality rate and time when GALM occurs at time $t_{d}$, before the maximum possible size has been reached (the mortality rate becomes infinite at time $\left.t_{\max }\right)$. Thus the mortality rate changes at GALM from $p_{n i}^{(1)}(t)$ (open circle) to $p_{n i}^{(2)}(t)$ (filled circle). The relation between noise mortality and the time of GALM, $p_{n i}^{(2)}\left(t_{d}\right)$, is shown as the thin broken line. The dotted line shows the size-independent mortality rate, $p_{n i}^{0}$. Parameter values are the same as the default values used for Figs. 2, 3 and 4, i.e. $p_{n i}^{g}=0.001, p_{n i}^{0}=0.01, g_{i}=0.1, W_{0}=1$, $W_{m}=10\left(t_{m}=90\right)$, and here we set (arbitrarily) $t_{d}=70$. In (a) and (b), the mortality rate $p_{n i}^{(1)}(t)$ increases linearly with time $t$ during growth from $W_{0}$ to $W_{d}$ (where $W_{d}<W_{m}$ ), following Eq. (3). If growth is arrested when parasite size reaches size $W_{d}$ at time $t_{d}$, the mortality rate changes to $p_{n i}^{(2)}$, following Eq. (7). In (c) and (d) the mortality rate $p_{n i}^{(1)}(t)$ increases linearly with time $t$ during growth from $W_{0}$ to $W_{d}$ (where $W_{d}<W_{m}$ ), following Eq. (4). If growth is arrested when parasite size reaches size $W_{d}$ at time $t_{d}$, the mortality rate changes to $p_{n i}^{(2)}$, following Eq. (8). GALM causes the size-dependent component of mortality to change by factor $\alpha$ (see Eqs. (7) and (8). In (a) and (c), $\alpha=0.2$, so that mortality rate decreases at GALM. In (a) and (c), $\alpha=1.5$, so that mortality rate increases at

\section{GALM.}


Fig. 2. The relation between fitness (lifetime egg production) and $W_{d}$ the size at GALM, between its lower limit of paratenicity $\left(W_{0}\right)$ and its upper limit where there is no GALM, but the larva continues to grow until it dies $\left(W_{m}\right)$. Each curve corresponds to a different value for the factor $\boldsymbol{\alpha}$ (values shown on each curve), which scales the size-dependent component of mortality rate at GALM. Filled circles show the unique or the higher fitness peak for each curve, open circles the lower peak when one exists. Parameter values are the same as those used for Figs. 3 and 4, i.e. $p_{n i}^{g}=$ $0.001, p_{n i L}^{0}=0.01, p_{j}=0.01, p_{i j}=0.02, g_{i}=0.1, g_{j}=0.2, W_{0}=1, W_{m}=10$. (a) the linear mortality model, (b) the accelerating mortality model. In (b) the open square indicates the fitness achieved by continuous growth (no GALM); this may be either a unique peak, or the higher or lower of two local optima depending on the value of $\alpha$. Curves are cubic splines fitted using the software package proFit to points iterated at $W_{d}$ intervals of 0.1 (i.e. 90 points per curve).

Fig. 3. Some effects of varying the parameters in the linear model (Eqs. (3) and (7)) on the prediction for optimal growth strategy in an intermediate host $\mathrm{i}$, in relation to the factor $\alpha$, which scales the size-dependent component of mortality rate at GALM. The optimal growth strategy may vary between immediate growth arrest at size $W_{0}$ (the size at infection, i.e. the paratenic strategy), or growth to GALM at size $W_{d}^{*}<W_{m}$, or continuous growth until death up to or before size $W_{m}$ (i.e. no GALM). Default parameter values are as in Fig. 2. (a) Variation in the parameter, $p_{n i}^{g}$, the parameter that scales the cost of size-dependent component of noise mortality. (b) Variation in $p_{j} / g_{j}$, the ratio: mortality rate in $\mathrm{j} /$ growth rate in $\mathrm{j}$. (c) 
Variation in $g_{i}$, the growth rate in i. (d) Variation in $\gamma=p_{n i L}^{0}+p_{i j}$, the sum of random (time-independent) mortality rate in $\mathrm{i}$, and transmission rate from $\mathrm{i}$ to $\mathrm{j}$.

Fig. 4. As Fig. 3, but for the accelerating mortality rate model (Eqs. (4) and (8)). Default parameter values are as in Fig. 2. 
Figure 1a:

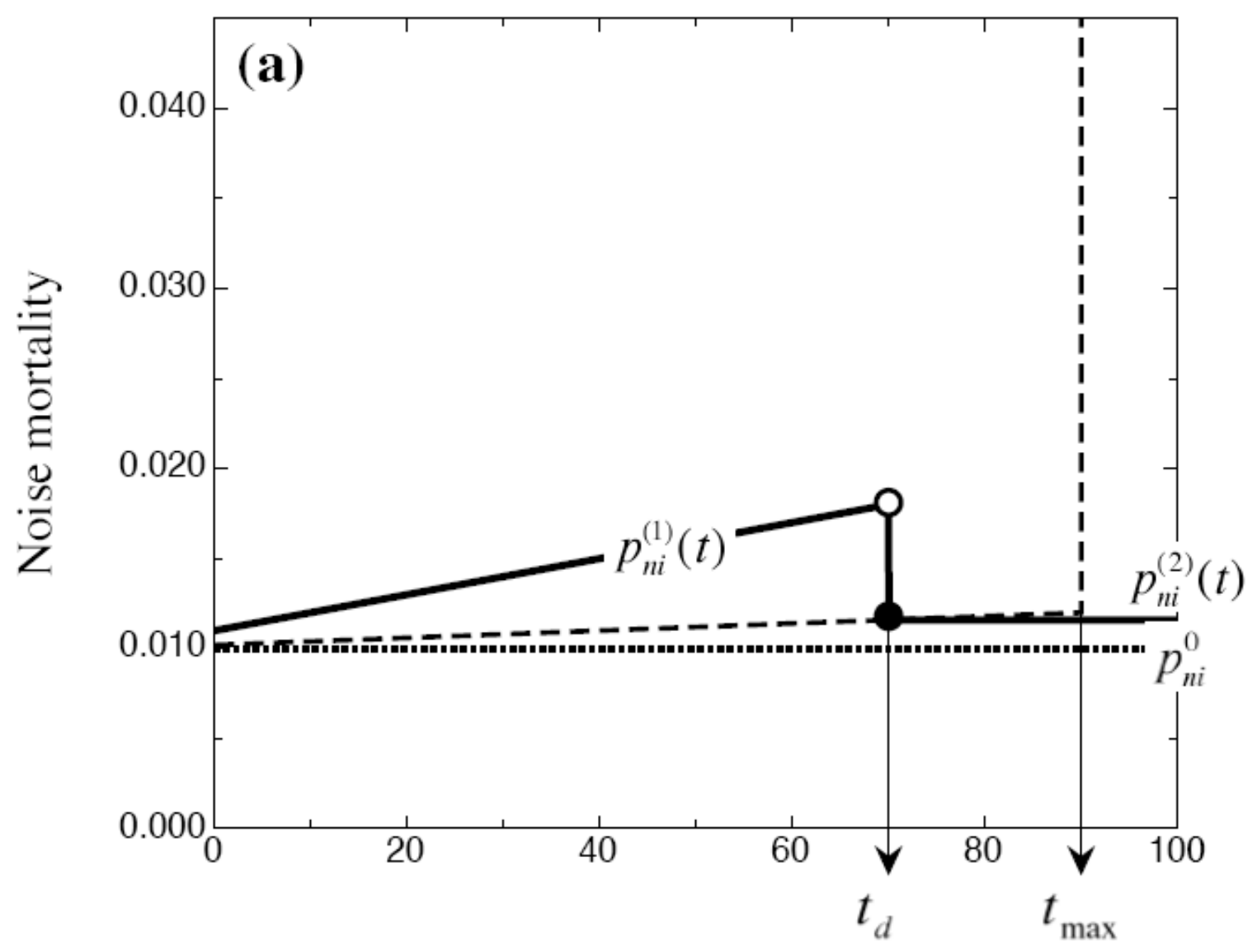

Time spent in host i, $t$ 
Figure 1b:

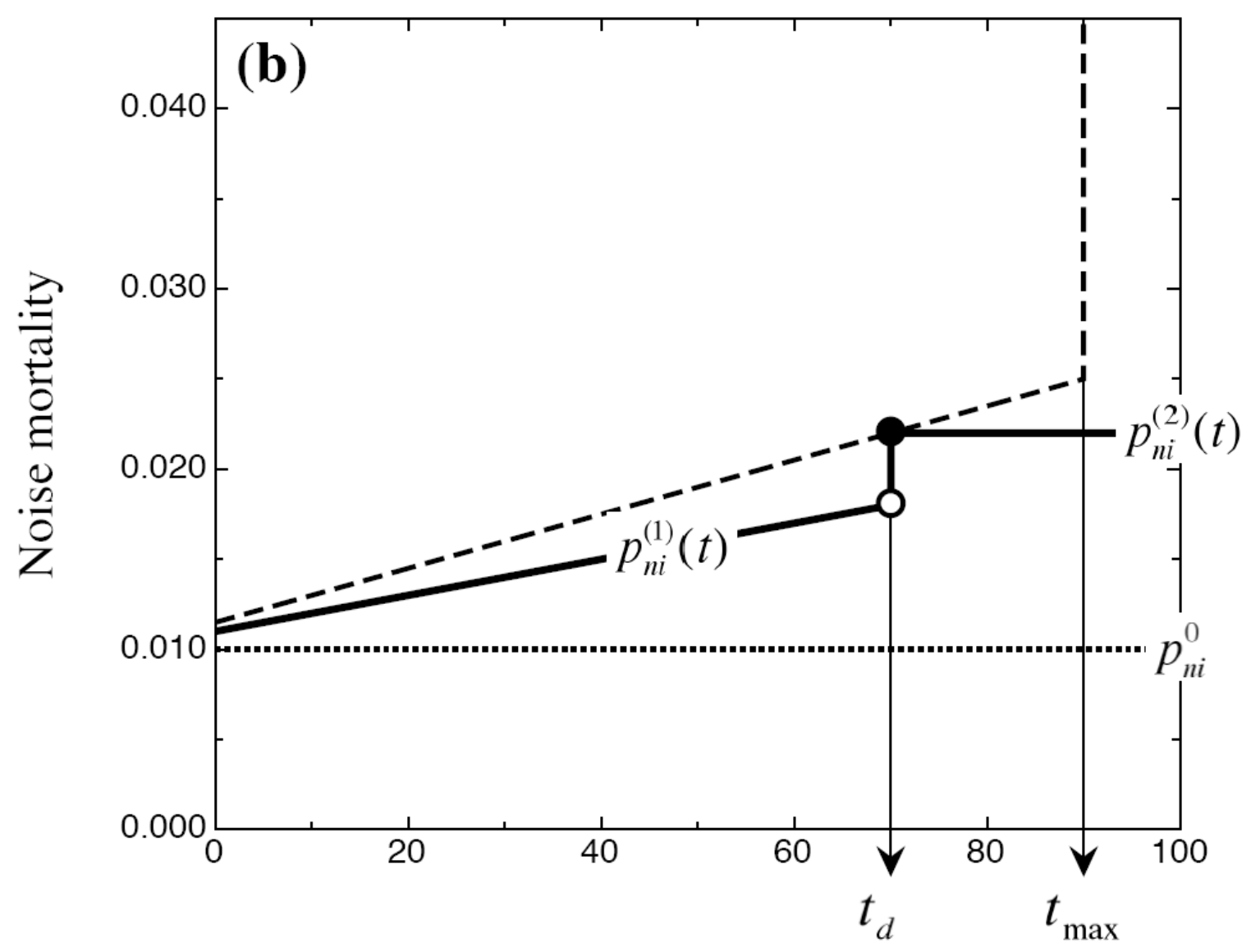

Time spent in host $\mathrm{i}, t$ 
Figure 1c:

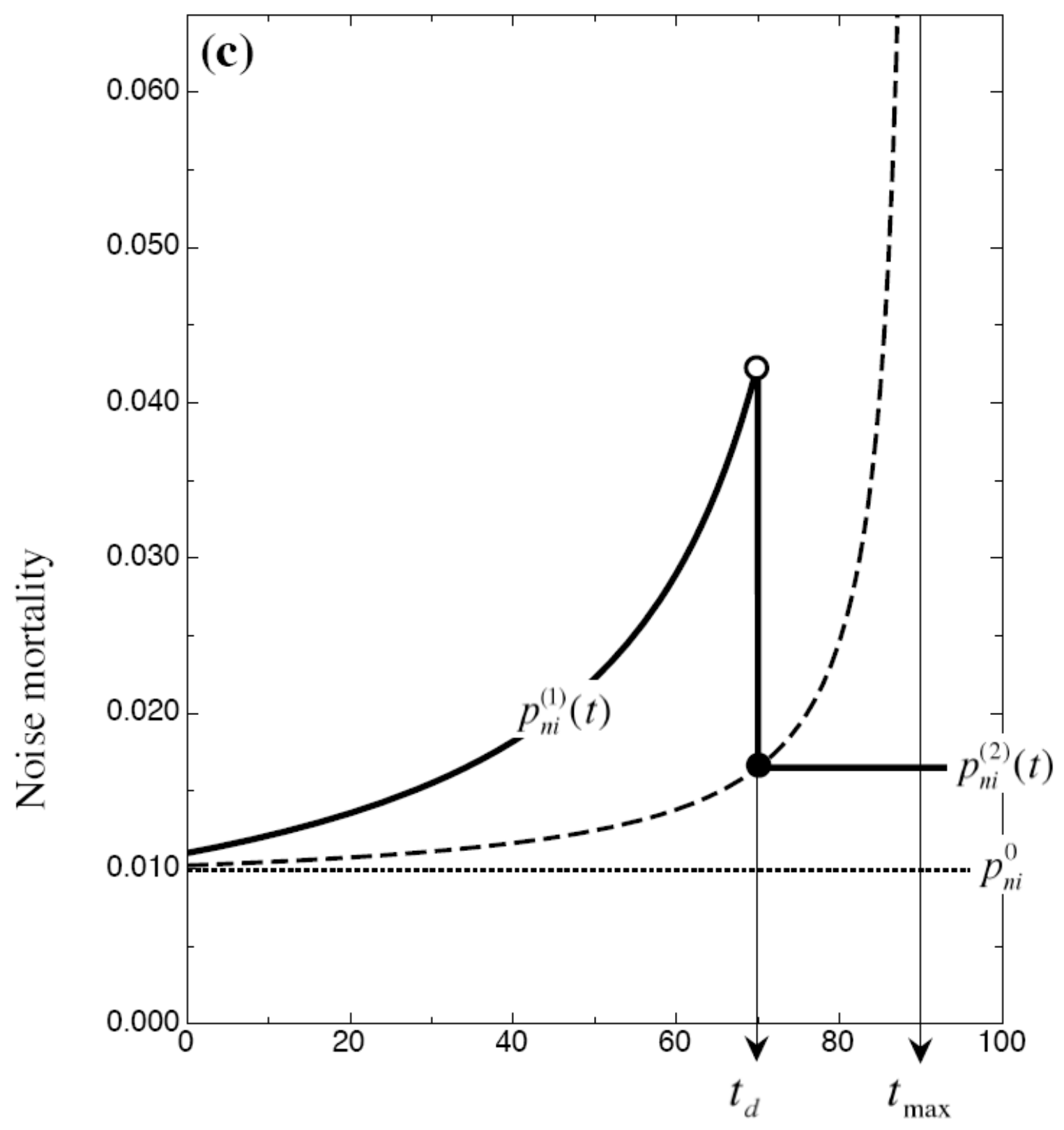

Time spent in host $\mathrm{i}, t$ 
Figure 1d:

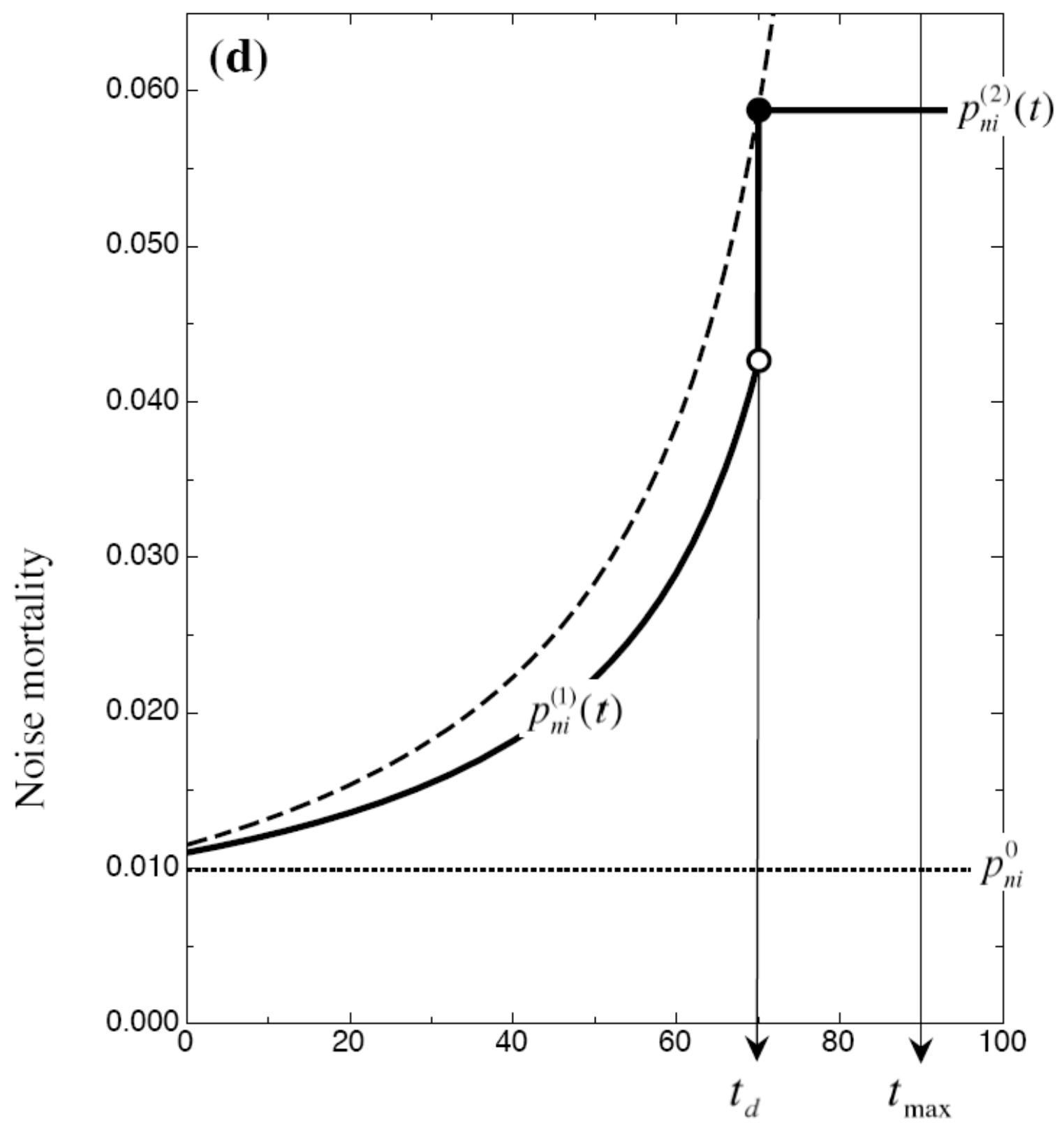

Time spent in host $\mathrm{i}, t$ 
Figure 2a:

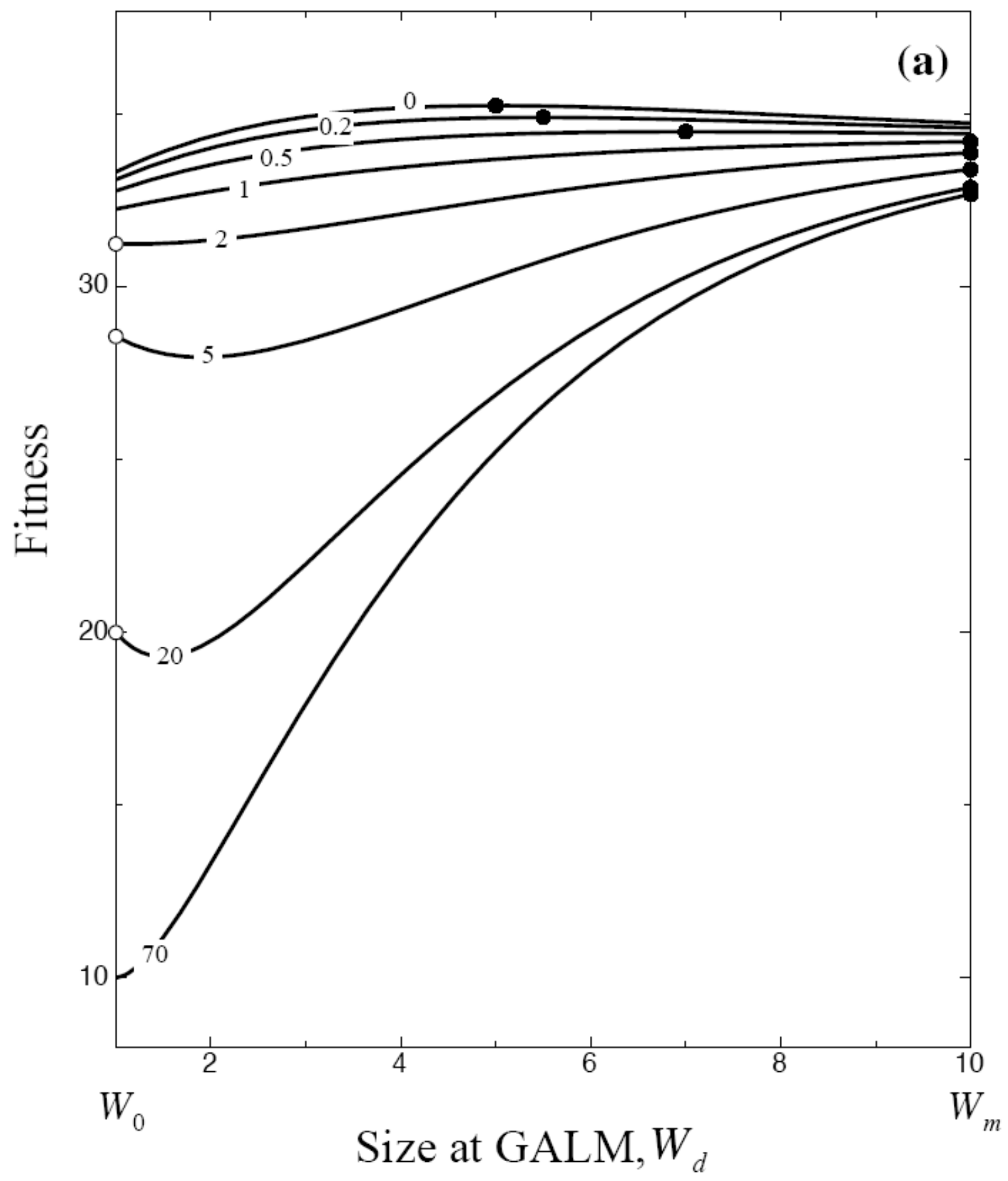


Figure 2b:

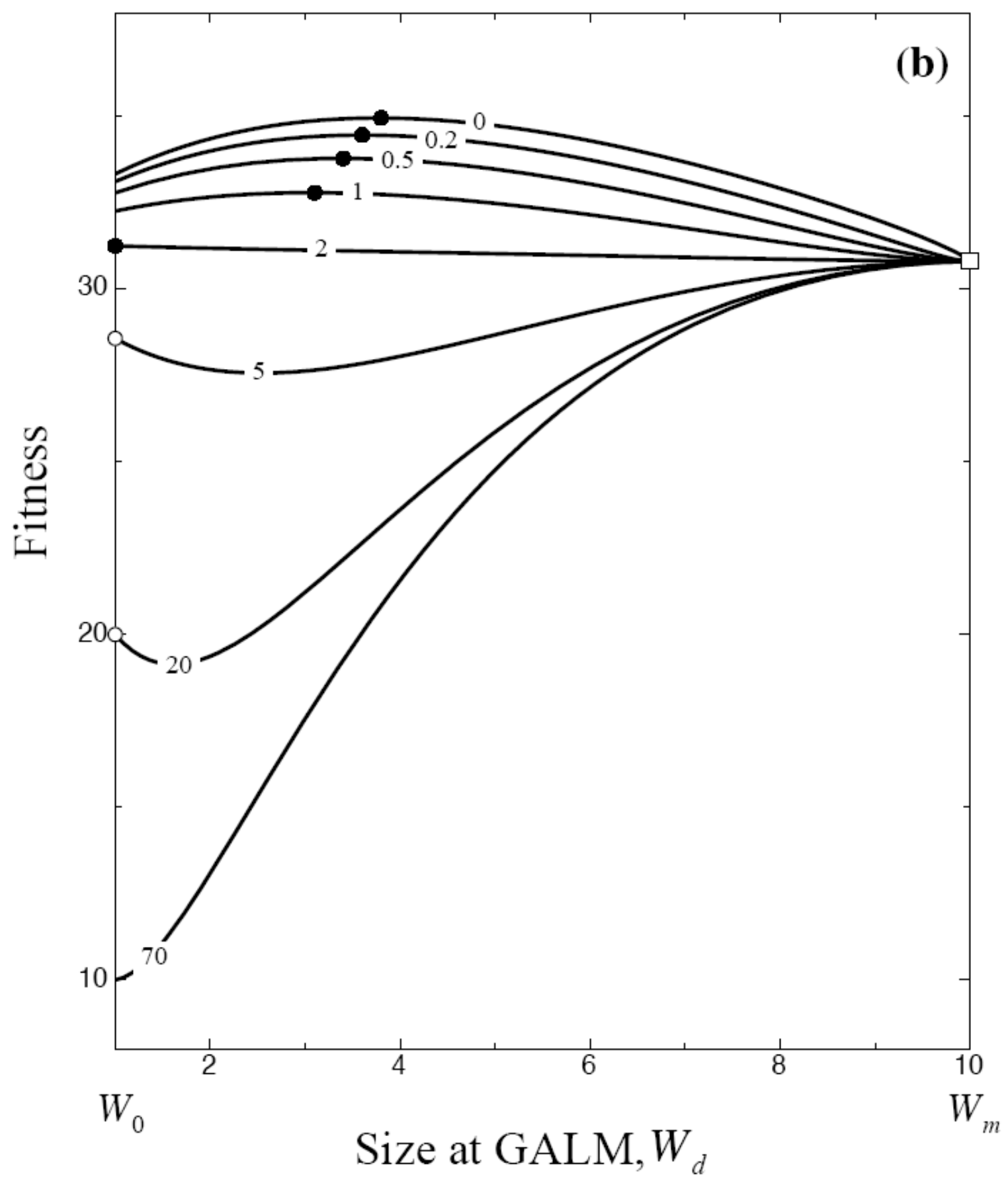


Figure 3a:

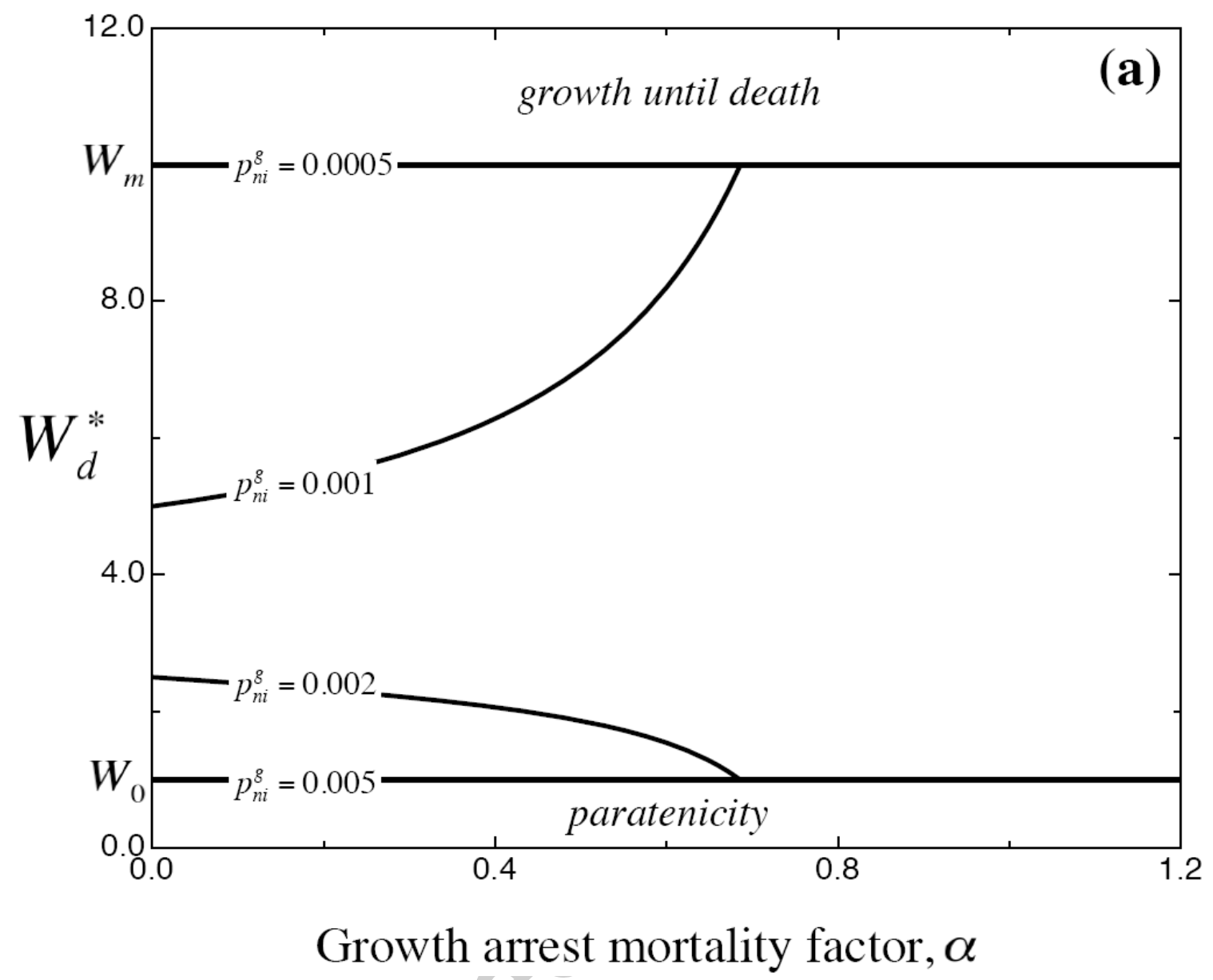


Figure 3b:

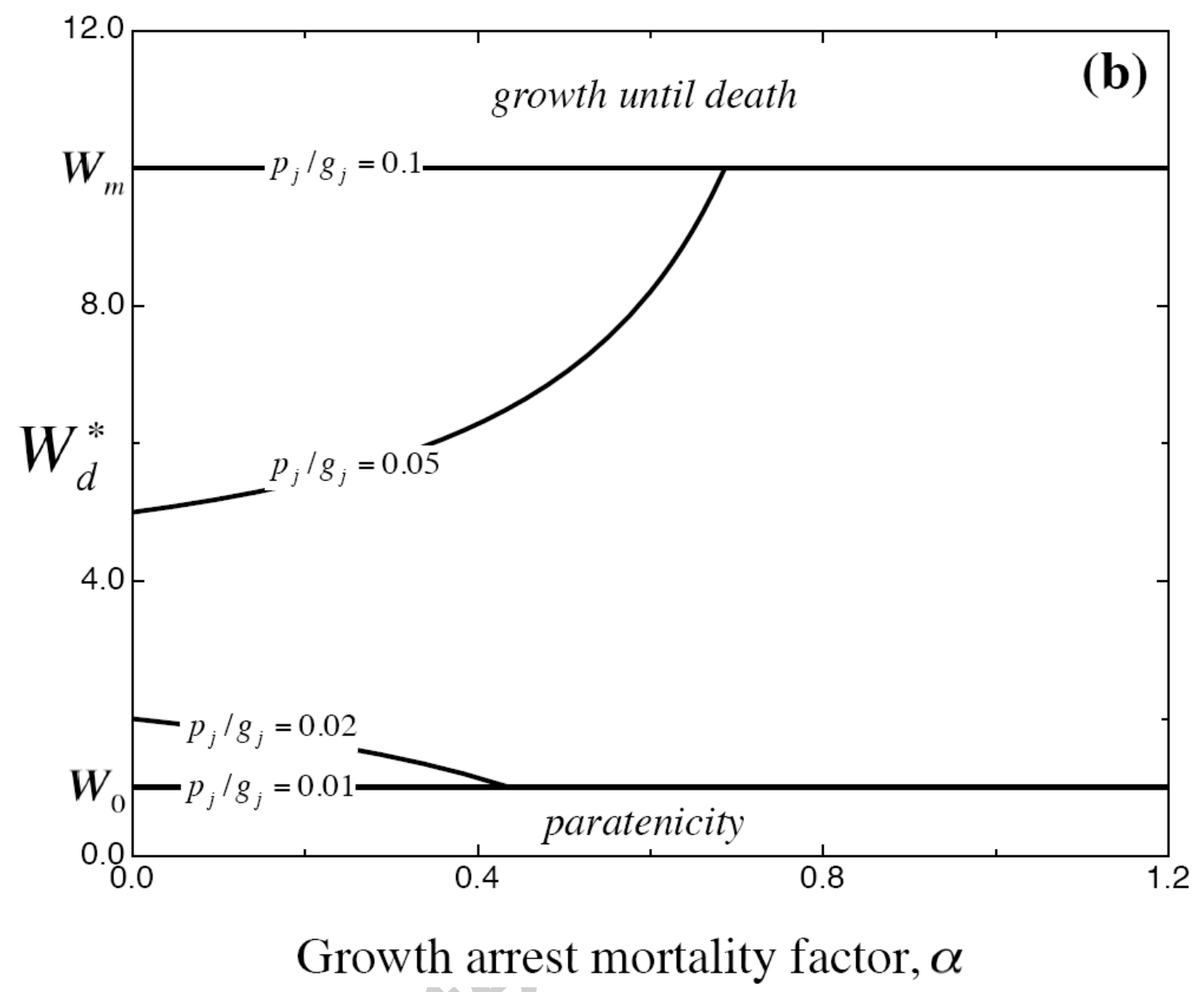


Figure 3c:

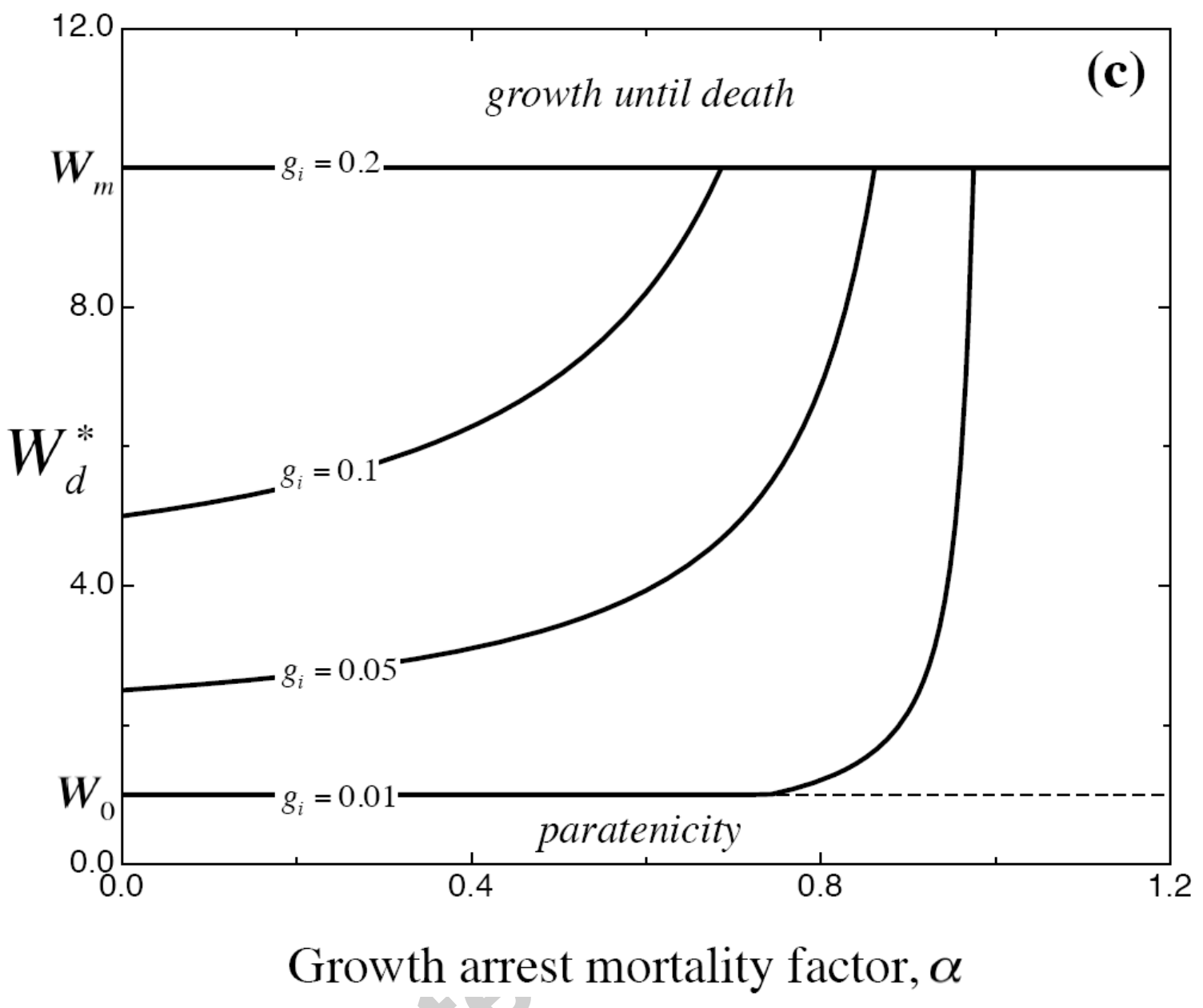


Figure 3d:

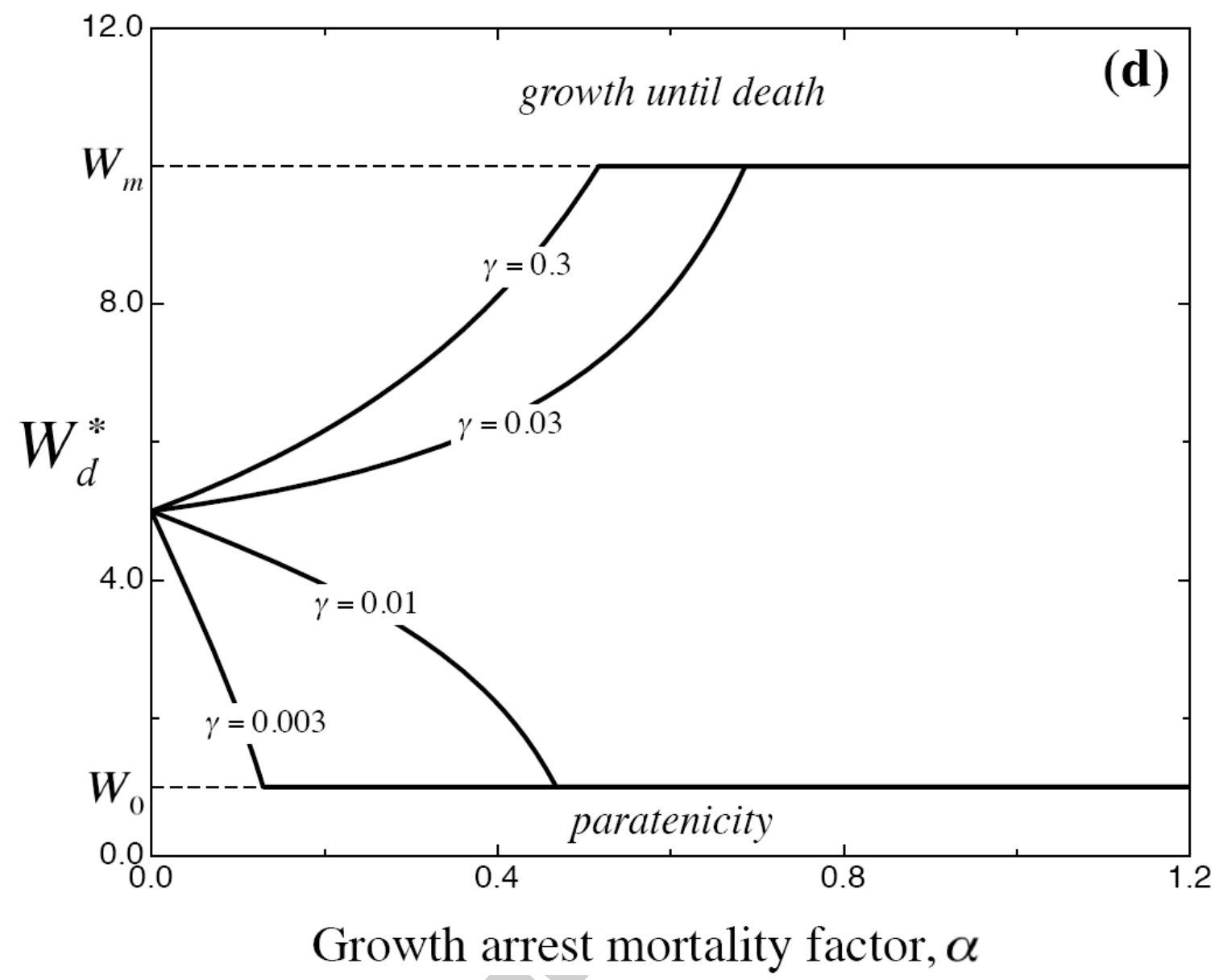


Figure 4a:

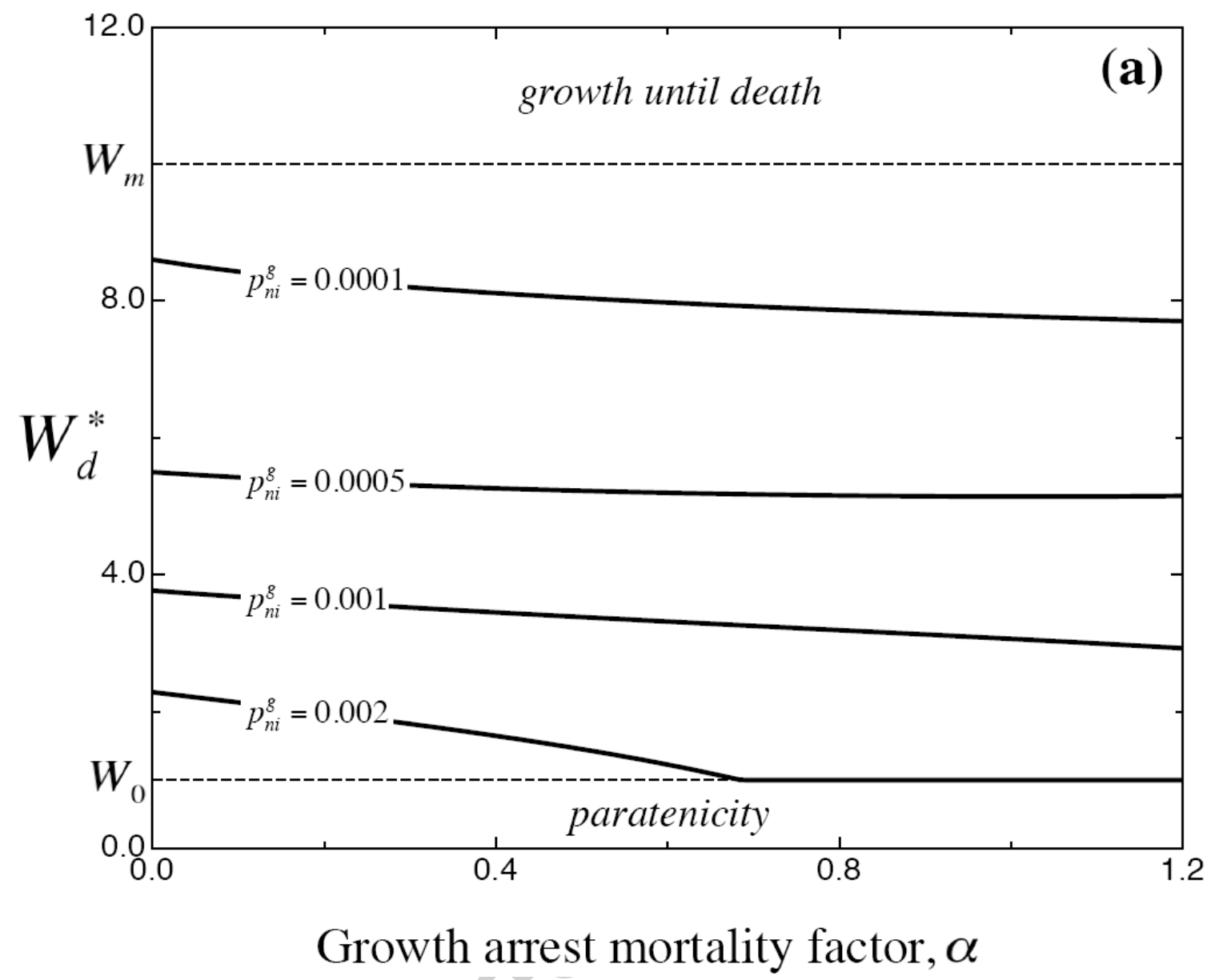


Figure 4b:

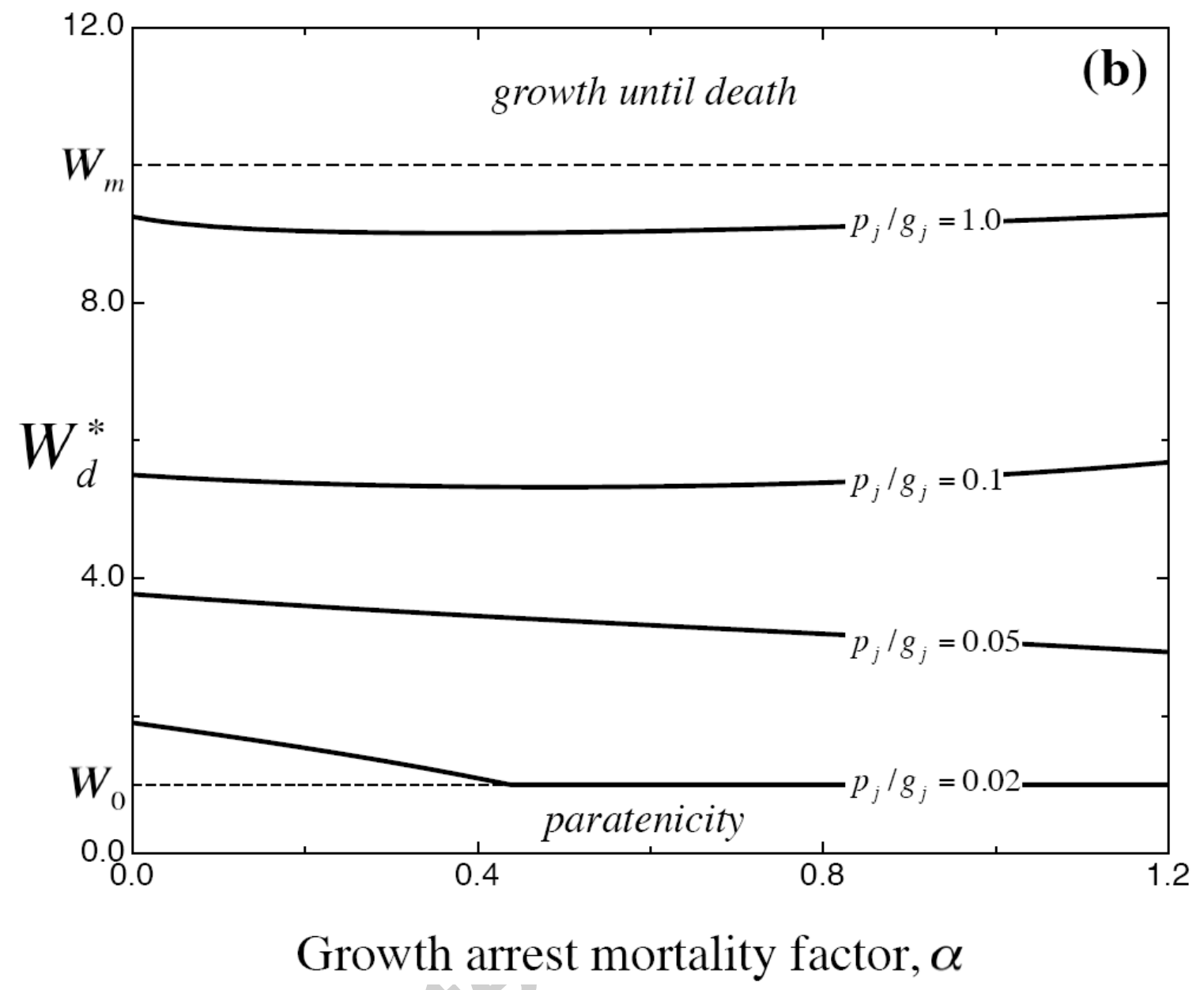


Figure 4c:

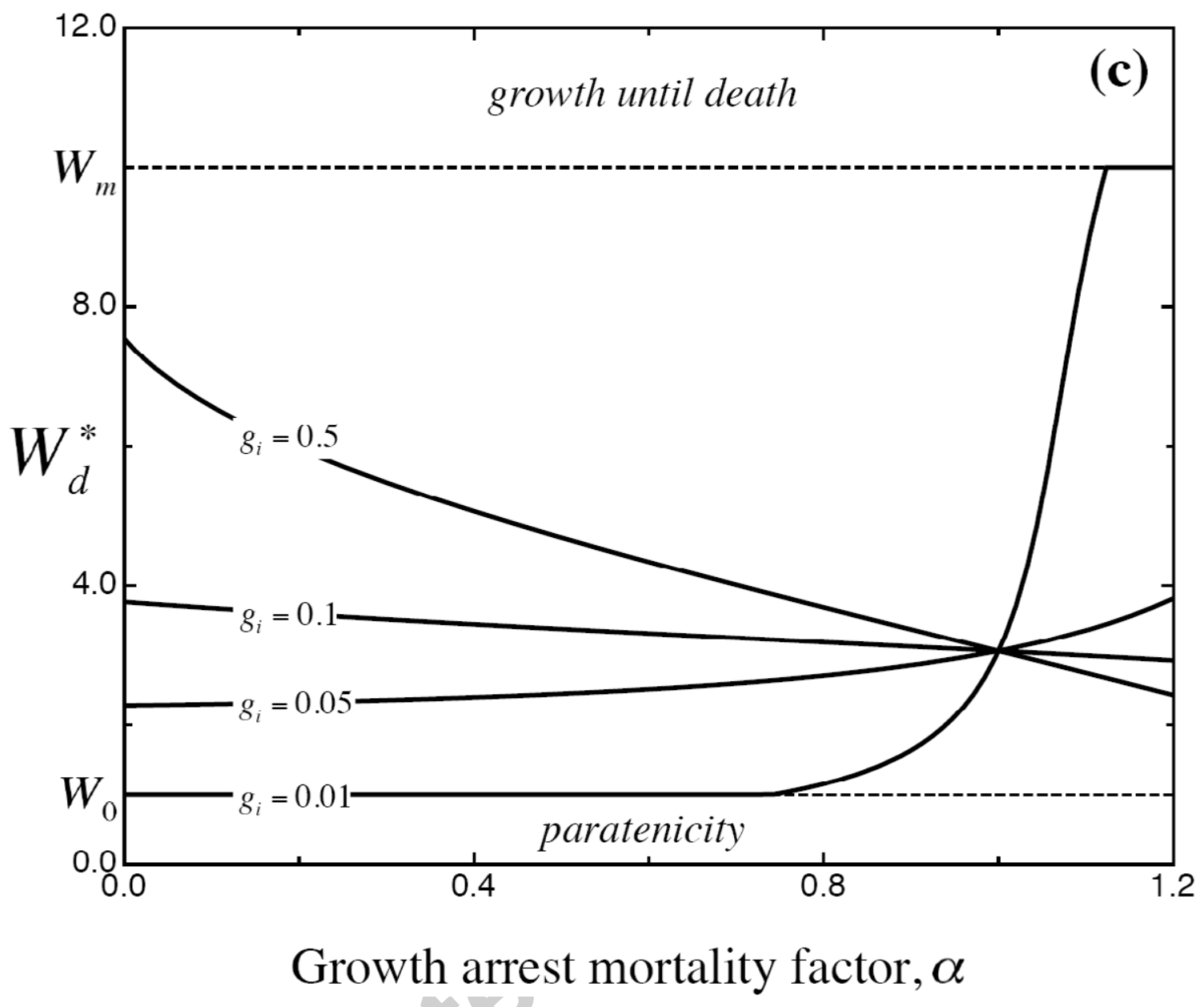


Figure 4d:

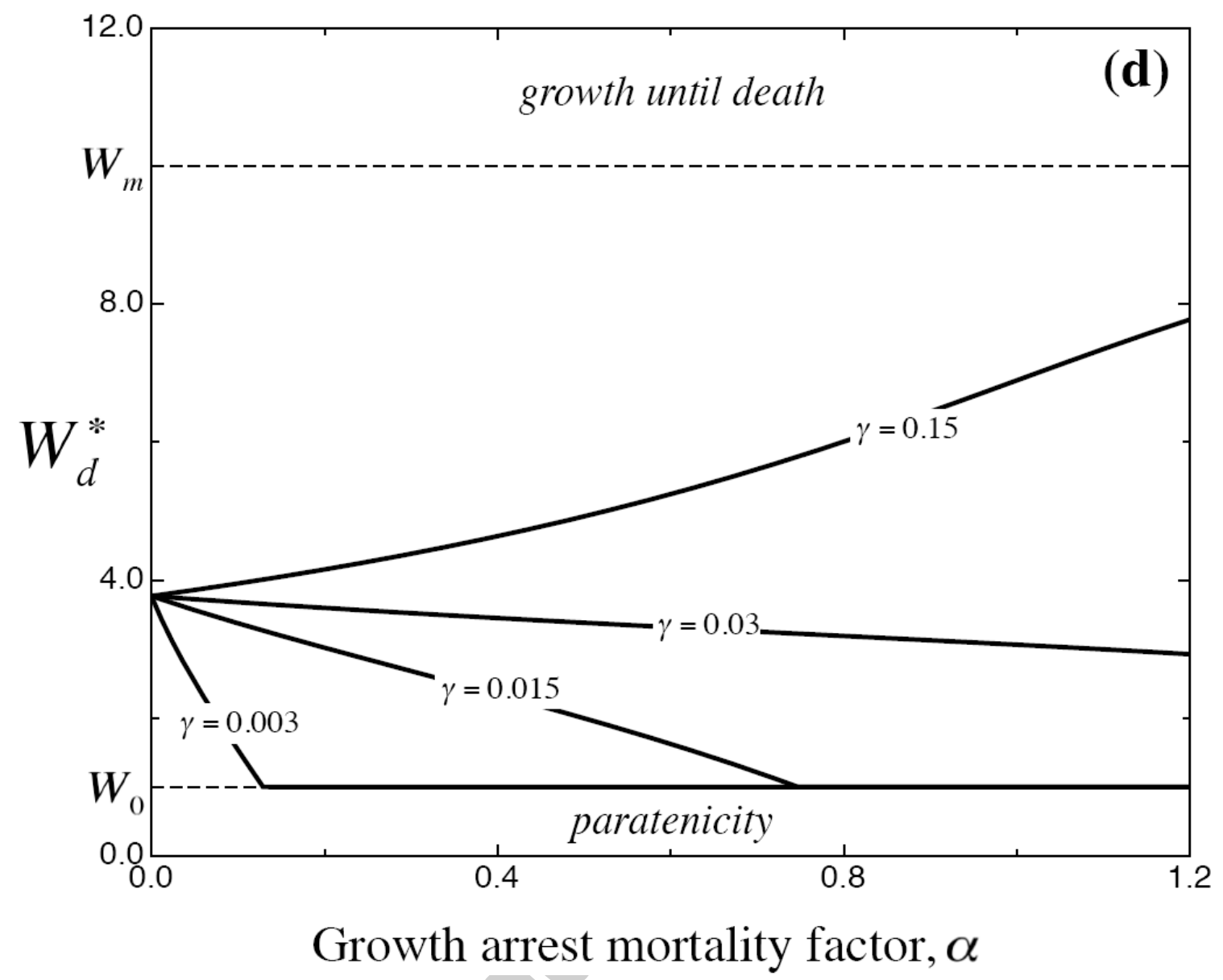

